\title{
Nomarski images for learning the anatomy, with tips for mosaic analysis*
}

\author{
John Yochem ${ }^{\S}$, Department of Genetics, Cell Biology, and \\ Development, University of Minnesota, Minneapolis MN 55455 USA
}

\section{Table of Contents}

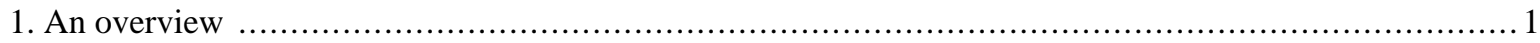

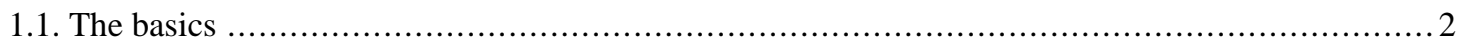

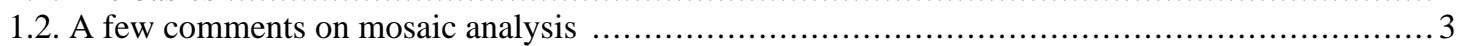

1.3. A few technical details on mosaic analysis ....................................................6 6

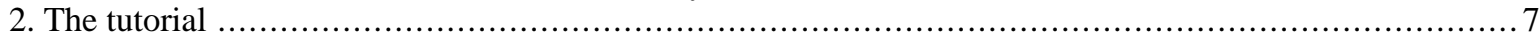

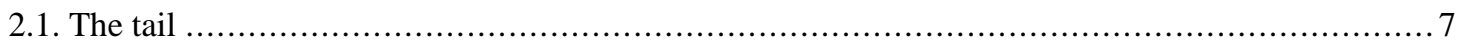

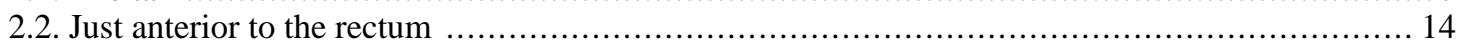

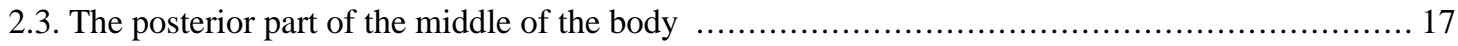

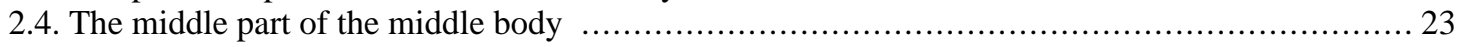

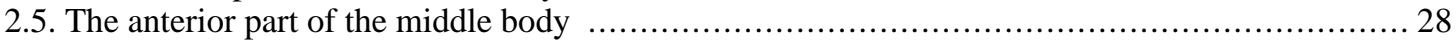

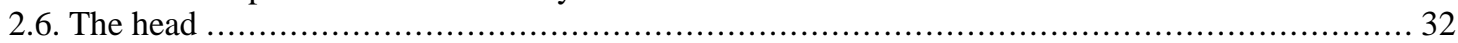

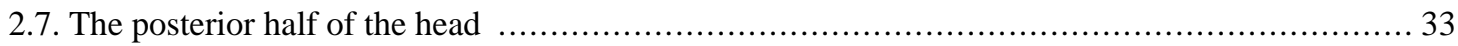

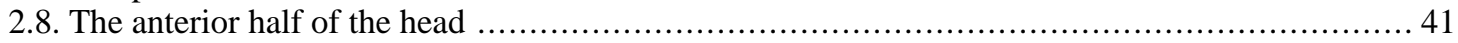

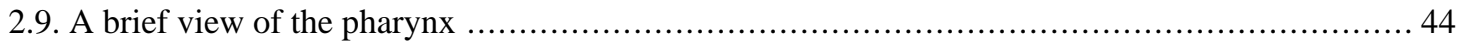

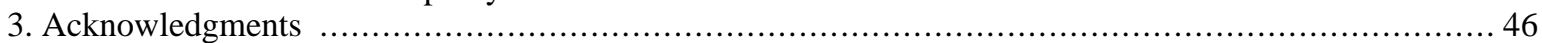

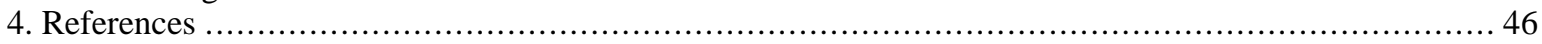

\section{An overview}

Knowledge of the positions of nuclei and of how these nuclei arise during development can be of great help for studies of Caenorhabditis elegans. For an analysis of genetic mosaics (see Genetic mosaics), this knowledge is indispensable. This tutorial provides an introduction to the anatomy by means of micrographs that depict the relative positions of certain nuclei in hermaphrodites. Because many of the original drawings of the positions of nuclei are dense with nuclei, the intent here is to present several focal planes for each of several regions of the animal. For simplicity, only a few "landmarks" will be emphasized. By landmarks, we mean certain cells whose nuclei usually can be discerned unambiguously in worms of more or less normal morphology. These landmarks can be learned with a little practice and, once committed to memory, can be used as guides for learning the relative positions of many other nuclei. The basis for this more advanced learning is drawings in the original publications of the cell lineage (Sulston and Horvitz, 1977; Kimble and Hirsh, 1979; Sulston et al., 1983) and at various computer sites (WormAtlas).

\footnotetext{
*Edited by David Fay. WormMethods editor, Victor Ambros. Last revised May 14, 2005. Published January 24, 2006. This chapter should be cited as: Yochem, J. Nomarski images for learning the anatomy, with tips for mosaic analysis (January 24, 2006), WormBook, ed. The C. elegans Research Community, WormBook, doi/10.1895/wormbook.1.100.1, http://www.wormbook.org.

Copyright: (C) 2006 John Yochem. This is an open-access article distributed under the terms of the Creative Commons Attribution License, which permits unrestricted use, distribution, and reproduction in any medium, provided the original author and source are credited. ${ }^{\S}$ To whom correspondence should be addressed. E-mail: yoche001@umn.edu
} 
Before beginning, some general words are in order. The tutorial is based on observations of N2 hermaphrodites (males are beyond the scope of the present endeavor) with differential-interference contrast (Nomarski) microscopy, which allows facile visualization of nuclei, because of their high refractility (Sulston and Horvitz, 1977). Lateral sections will be presented, which is standard in C. elegans and will correspond with many of the drawings of nuclei in the original publications. The design was to provide an image of the part of the anatomy that one wishes to learn, which can be viewed on the computer monitors of the microscope as one goes through the focal planes of a living worm until a match is seen in side-by-side images.

Limitations of time mean that not all of the potential landmarks will be indicated, but we hope to add more later. Also, caution is in order when venturing beyond the landmarks. Some parts of the anatomy are challenging and require careful study, and it is always wise to consult someone when in doubt. In the tutorial, when we mention without attribution the position, migration, lineage, or differentiation of a cell, that knowledge is based on the analyses of the cell lineage (Sulston and Horvitz, 1977; Kimble and Hirsh, 1979; Sulston et al., 1983). The diagrams in one reference have been particularly useful (Sulston and Horvitz, 1977), and one may also want to examine diagrams in "The Mind of a Worm" (White et al., 1986). We hope that the present endeavor extends these works by being a computer-based canvas that permits more micrographs than were originally practical, with computer links for students wishing a greater understanding of the function and implications of a particular cell or tissue.

\subsection{The basics}

It is important to realize that the morphology of your worm may differ from what we present. Not only can mutations and developmental defects affect the anatomy; natural variation from worm to worm is also to be expected, and specimens can become deformed during mounting onto slides. While discussing the tail, for example, we shall mention that the neurons DVB, DVA, and DVC form an arc in the central focal plane, but distinguishing the three in a particular worm-perhaps in many of them-may require adjustments of the focus, and an arc may not be present. (Stating that DVB, DVA, and DVC form an arc brings up another basic point. We mean, of course, that the nuclei of these cells form an arc. In most cases, the naming of a cell means the nucleus of that cell. It is easy to keep track of many cells, such as neurons and body muscles, because they are mononucleate. In cases of binucleate or syncytial cells, such as hyp10 and hyp7, we shall usually be referring to particular nuclei of those cells.

Differences in sex or developmental stage can also result in deviations from the images we show here. Most of the images will be of L3 or L4 hermaphrodites, but at one point, a difference between juvenile males and hermaphrodites will be shown. It should also be mentioned that extrachromosomal elements, whether arrays or free duplications, can adversely affect morphology; neither a $D p(+)$ worm nor a transgenic worm is a wild-type worm.

The micrographs are presented with anterior to the left and dorsal at the top. The rectum, the neurons of the ventral cord, and the nucleus of the excretory cell allow you easily to distinguish ventral from dorsal even in hatchlings. In older hermaphrodites, the vulva or its precursor is also useful. In most cases, we shall present focal planes of the left side, leaving it to the student to study the images on the right, which are usually mirror images of those on the left. We are presenting lateral (longitudinal) views, because a worm usually lies on its left or right side when mounted on agar pads for Nomarski microscopy (see Methods in cell biology). The left optical sections are presented in order to correspond to the typical diagrams in the original publications of the cell lineage mentioned above.

It is worthwhile from the start to become familiar with the left and right sides of the worm. If the anterior end of the worm is to your left and dorsal away from you as you look in the microscope, then the left side is up, and its right side down (Figure 1). That is, the worm is lying on its right side. If your image is of the central focal plane and you move the objective up, you will be going through the left side of that part of the animal. (As you move the objective through the right focal planes of a worm lying on its right side, you may notice that images are not as sharp as those from the left side of the animal.) Asymmetries in the worm make it fun to practice left versus right with the microscope. For example, the anterior arm of the gonad is on the right side of the intestine, and the posterior arm on the left (Figure 2). Also, a pair of ventral coelomocytes is on the right side of the animal; they can be found in the anterior part of the middle body somewhat near but posterior to the junction of the pharynx and intestine. Another pair of ventral coelomocytes is on the left; they are located just anterior to the vulva. 


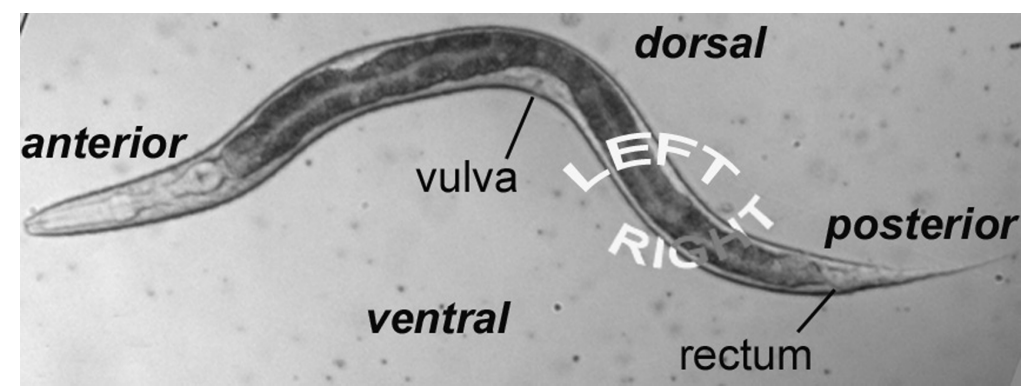

Figure 1. An L4 hermaphrodite lying on its right side.

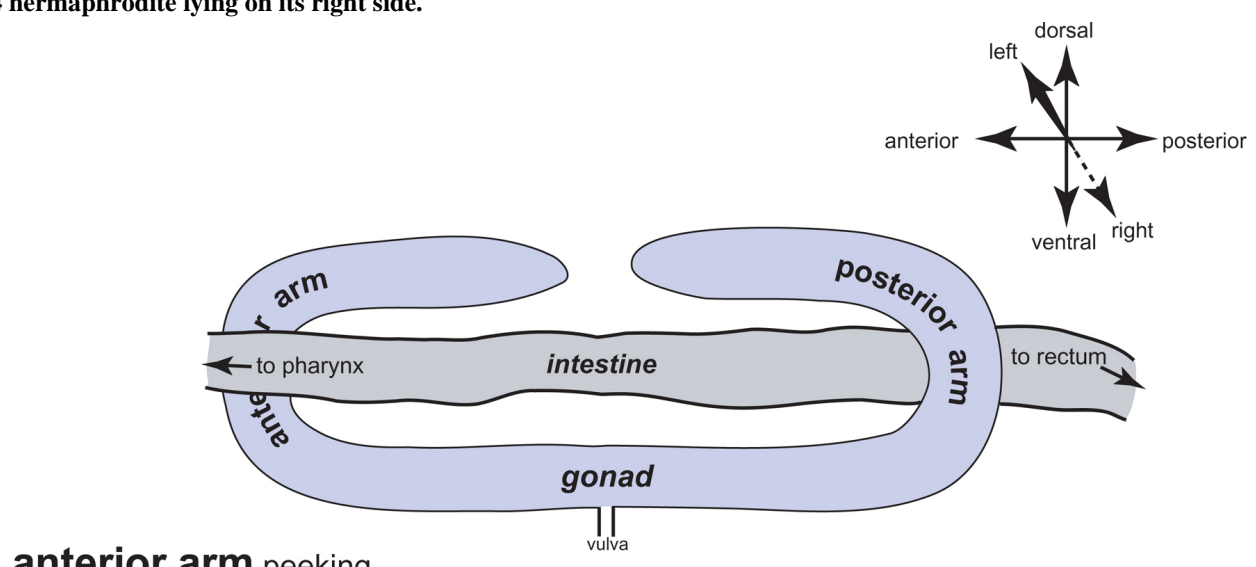

anterior arm peeking

out from behind intestine
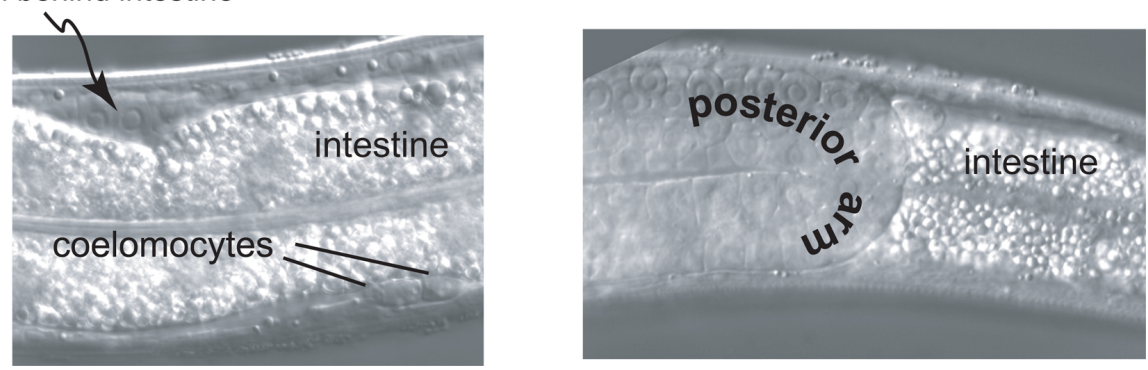

Figure 2. A highly exaggerated schematic of the left-right relationship of the gonadal arms with the intestine. A real image of the posterior arm is shown in the micrograph (lower right) of part of an L4 larva lying on its right side, such that left is towards you, and right is through the paper or computer screen. The posterior arm stands out in front of (to the left of, from the point of view of the worm) the highly vacuolated intestine. The gonadal arm is somewhat "transparent" with these optics, permitting some of the intestine to be seen through it. The corresponding region of the anterior arm is shown in the left micrograph. The highly refractile vesicles of the intestine completely obscure the gonadal arm behind (to the right of) it. A pair of coelomocytes that is located on an animal's left is also in view. This is not surprising, because the focal plane of the intestine in this view is left of the anterior arm of the gonad. With respect to the body at large, this pair of coelomocytes is present in the ventral part of the middle body, just anterior of the vulva.

We have just presented a view of a worm that is lying on its right side with its anterior end to the viewer's left. Of course, the worm next door may be lying on its left side, or it could be lying on its right side with its posterior to the viewer's left, and so forth. It is therefore worthwhile to learn to make the requisite adjustments in one's head during the microscopy.

We shall begin at the end of the worm. Although rich in cell types, the tail is less complicated than the head, and navigation is therefore more straightforward. Once familiar with the tail, we shall move anteriorly a few sections at a time. This approach should prepare us to tackle the head, with its many neurons, body muscles, and hypodermal nuclei-not to mention the pharynx.

\subsection{A few comments on mosaic analysis}

Mosaic analysis as typically practiced in C. elegans is based on the spontaneous mitotic loss of a free duplication or of an extrachromosomal array during embryogenesis (see Genetic mosaics). For simplicity, we shall use the term extrachromosomal element for both. Strains are constructed such that the element contains a functional 
copy of a gene, but both copies of the gene on the homologous chromosomes have a recessive mutation. A functional copy of the gene is therefore only present in a cell when that cell inherits a copy of the element. The point of mosaic analysis is, of course, to determine how cellular inheritance of a functional gene affects phenotype. A marker expressed by the element can be of great help in elucidating the cellular inheritance of the element. Because the anatomy for our purposes is based on the positions of nuclei, markers have been chosen that cell autonomously affect nuclei. For example, recessive mutations in the ncl-1 gene confer an enlarged nucleolus (Hedgecock and Herman, 1995), as shown in Figure 3 and Figure 4, where nuclei in the central longitudinal section of the tail are compared between wild type and the corresponding image from CB3388 [ncl-1(e1865)]. Constructs that express high levels of green fluorescent protein (GFP) in nuclei can also be used. One such marker, SUR-5::GFP, is useful for finding mosaics that had losses of an extrachromosomal array early in embryogenesis (Yochem et al., 1998).
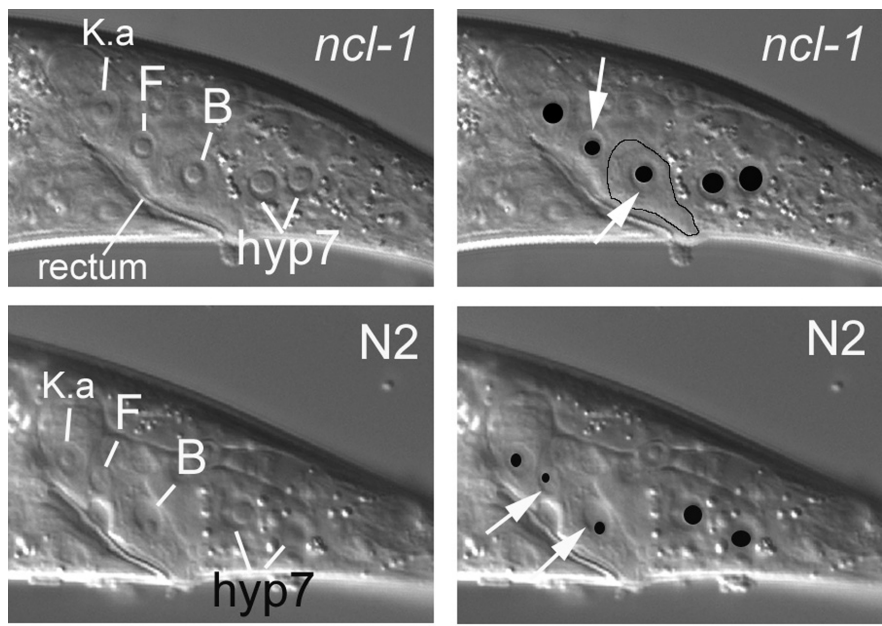

Figure 3. The Ncl phenotype. The top panels demonstrate enlarged nucleoli conferred by a recessive mutation in the $n c l-1$ gene. The images are from the central focal plane of the tail of a hermaphrodite, with the cells K.a, F, and B indicated. Each of the white lines ends at the boundary of a nucleus, which appears as a depression in these images. A nucleolus appears as a dome within the depression. In the top right panel, the same image is shown with the nucleoli overlaid with black ellipses and with white arrows demarcating an edge of the nucleus of either F or B. Although cell boundaries are often not apparent with Nomarski optics (recall that we are studying the positions of nuclei), the actual cell boundary of B is somewhat visible here and is therefore roughly outlined (except for rectal cuticle on the apical side of B). The normal nucleoli of these cells in a wild-type animal are shown in the lower panels. Note how much smaller the domes are-how much less area of each nucleus is occupied by the nucleolus.
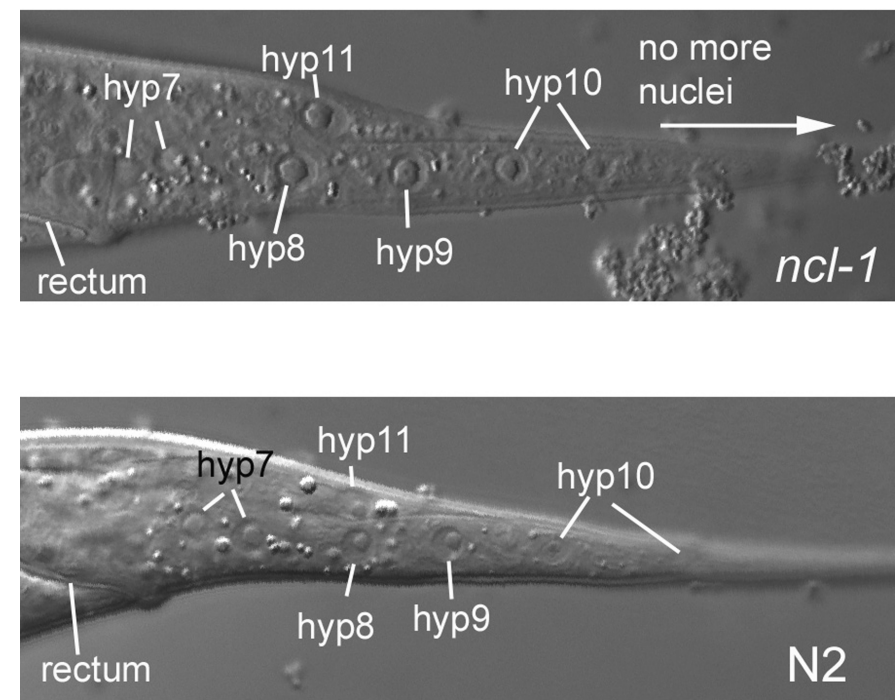

Figure 4. Another image of the Ncl phenotype. The nucleoli of the hypodermal cells hyp8, hyp9, hyp10, and hyp11 stand out in this image of the central focal plane of the tail from an L4 hermaphrodite homozygous for $n c l-1(e 1865)$. The corresponding wild-type image from an N2 hermaphrodite is shown below for comparison. The posterior hyp10 nucleus is not completely in the focal plane. This is a reminder that adjustments of the focus are almost always required in real life. We should also remind you that we are presenting only lateral views, so these are from the central focal plane of longitudinal sections through the animal. 
A cell that fails to inherit the element lacks a wild-type copy of the gene. If this cell divides, its descendants, of course, also lack a wild-type copy of the gene. Loss of an element in a progenitor cell therefore produces a negative clone of cells with respect to the gene. It should be mentioned here that the word "clone" is a bit inaccurate. The term really should be used in cases where the progenitor produces identical copies, as has been discussed (Kimble and Hirsh, 1979). Here we shall take some license and continue to use the word clone. In fact, we shall mention both positive and negative clones. Positive clones are appreciated when using a fluorescent marker such as SUR-5::GFP, because the descendants of the positive progenitor fluoresce against a dark background. Because of the ability to examine rapidly many hundreds of animals with a dissecting scope equipped with an $\mathrm{Hg}$ or halogen lamp for fluorescence, one can easily find rare animals that have had sequential losses of an extrachromosomal element. In the case of sequential losses, the clonal descendants can have increased brightness, probably because the failure to segregate the element earlier in the cell lineage resulted in an increase in copy number of the element in the progenitor cell and its descendants. An increase in copy number of the element would, of course, also mean an increase in copy number of the marker gene. Sequential losses can be informative. For example, mosaics have been seen in which the only positive clone was established by the blastomere C. That is, only the descendants of $\mathrm{C}$ fluoresce. Because $\mathrm{C}$ contributes nuclei to the large syncytium hyp7 that composes most of the hypodermis, such positive clones can be of use in investigating whether or not hyp7 might be a focus of activity of a gene (Moribe et al., 2004).

Implicit in the foregoing is the necessity for knowing where in the animal the various descendants of a progenitor cell reside. The nature of the cell lineage and anatomy is such that cells related by lineage may not end up near each other. Throughout this tutorial, we shall try to point out the usefulness of a cell for mosaic analysis, but we will not always be able to do this because of limitations at this time. In fact, we are the first to admit that the tutorial is woefully inadequate in one of its intended purposes, the explaining of the anatomy in terms of the cell lineage for mosaic analysis. For now, we shall only briefly mention the lineage of a handful of cells and their usefulness for mosaic analysis. This deficiency is not, however, catastrophic, because once one becomes comfortable with the anatomy and the lineal relationships of certain cells, one can gain additional insight by consulting the "parts lists" and lineage diagrams in the original publications cited above.

Knowledge of the cell lineage is also important in that some cells have more than one nucleus. These nuclei can descend from different parts of the cell lineage and therefore can cause confusion during mosaic analysis. Hyp10, for instance, is binucleate, and the aforementioned hyp7 is composed of many nuclei, some of which descend from $\mathrm{AB}$ and some from $\mathrm{P} 1$ (Figure 5). Because $\mathrm{AB}$ and $\mathrm{P} 1$ are the daughters of the zygote, hyp7 cannot be distinguished by the loss of an extrachromosomal element during the first embryonic division. Nevertheless, although an analysis of the hyp7 syncytium is challenging, it can be implicated as the focus of the activity of a gene (Moribe et al., 2004; Myers and Greenwald, 2005). Here and there, we shall try to make note of some of the cells that have more than one nucleus. We shall also make an effort to point out plasticity in the cell lineage that can cause confusion. Two examples are the vulval precursor cells and a pair of hypodermal cells in the tail called hyp 8 and hyp9. 


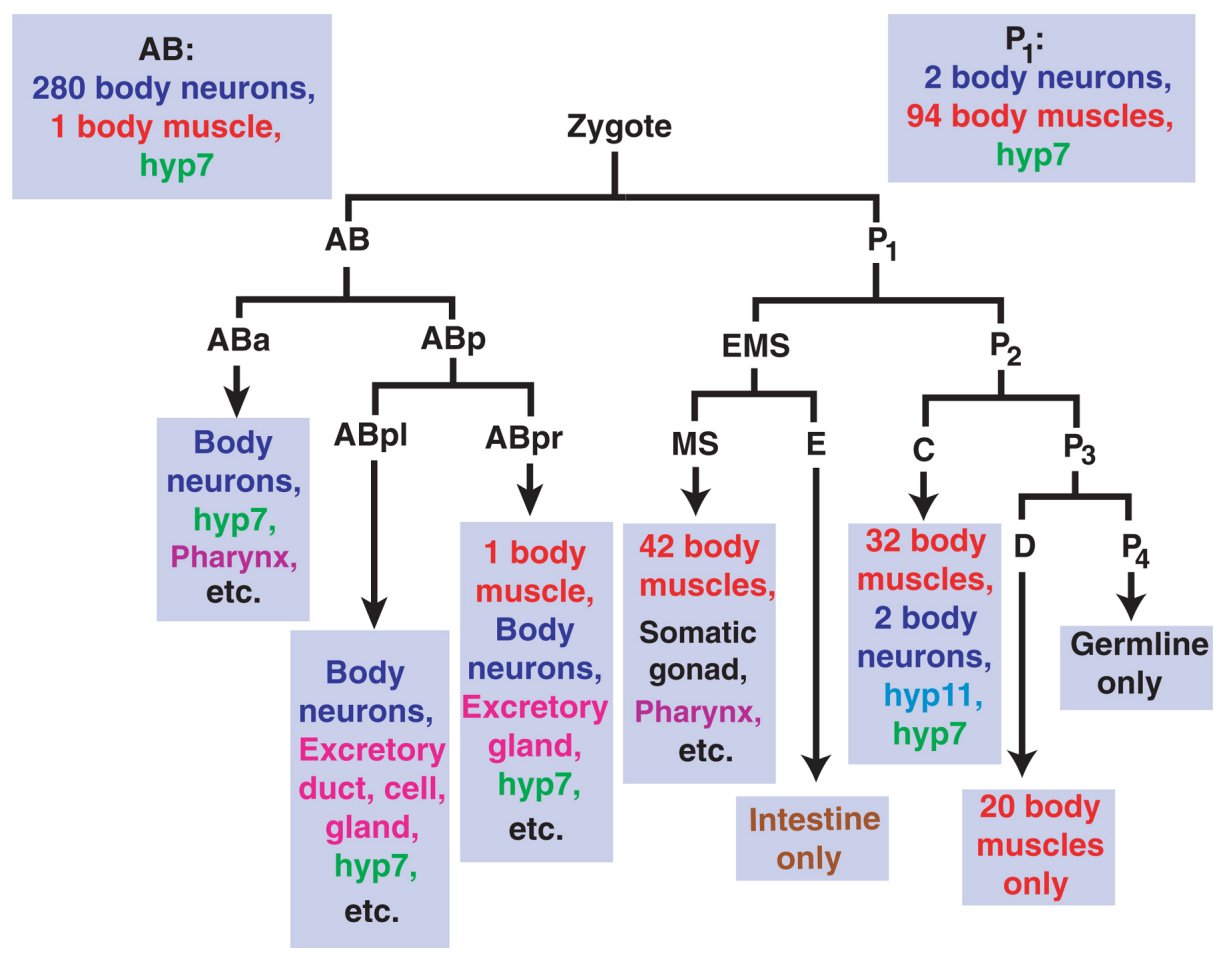

Figure 5. The embryonic progenitors of several cell types in a hermaphrodite. The blastomere D only produces 20 body muscles. E is the progenitor of all of the intestinal cells, and P4 is the progenitor of all of the germ cells. C is the progenitor of four cell types: neurons (a total of two), body muscles (a total of 32), the hypodermal cell hyp11, and part of the large syncytium hyp7 (descendants of AB also contribute to the formation of hyp7). Additional cell types derive from $\mathrm{ABa}, \mathrm{ABpl}, \mathrm{ABpr}$, and MS. For instance, $\mathrm{ABa}$ and $\mathrm{MS}$ are the progenitors of the pharynx, and the somatic gonad descends from MS. For more details of the cell lineage, see Wormatlas.

\subsection{A few technical details on mosaic analysis}

Although knowledge of the anatomy and cell lineage is a prerequisite for mosaic analysis, there are also technical details that are useful to know. Markers such as unc-36 and SUR-5::GFP make it possible to use a dissecting scope to screen worms on a normal Petri plate for those having certain losses. For detailed analyses of mosaicism, one must, however, turn to higher magnifications. The following four paragraphs are modifications of several details concerning the search for and microscopy of genetic mosaics (Yochem et al., 2000). Originally published in Developmental Biology Protocols I (Methods in Molecular Biology, vol. 135), edited by R. Tuan and C. Lo, the details are reprinted with permission of the publisher.

In some cases, mosaic analysis can be greatly accelerated if it is possible to examine hundreds of animals at once for those rare worms that have had early losses of an extrachromosomal element. One approach is a cell lineage marker such as unc-36. This gene must be expressed in descendants of ABp for normal, coordinated movement (Kenyon, 1986). If $\mathrm{AB}$ or $\mathrm{ABp}$ fails to inherit a wild-type copy of the gene, the animals are nearly paralyzed; losses in $\mathrm{ABa}$ or in the $\mathrm{P}_{1}$ side of the cell lineage do not confer the unc-36 phenotype. Other cell lineage markers are listed in Table 3 of the article cited above. If the element expresses SUR-5::GFP, one can examine hundreds of worms at once with a fluorescence dissecting microscope for those worms having mosaic patterns of green fluorescence (Yochem et al., 1998). For example, if you find a mosaic in which all of the nuclei of the intestinal cells fail to fluorescence, the animal has had a loss in either $\mathrm{P}_{1}$, EMS, or E. This conclusion is evident from studying the diagram of the lineage: all of the intestinal cells derive from E; and EMS and $\mathrm{P}_{1}$ are themselves precursors of $\mathrm{E}$ (Figure 5). It is possible with the dissecting microscope to make a good guess as to which of these is the point of loss. A loss in EMS, for instance, would affect the uterus and a subset of the body muscles. A loss in $\mathrm{P}_{1}$, on the other hand, would be even more extensive and would affect all of the body muscles save for one.

Another detail to keep in mind is that an animal can have more than one loss. Independent losses are especially likely when using extrachromosomal arrays, even if the rate of loss is within what is generally accepted as suitable for mosaic analysis, typically $0.1 \times 10^{-3}$ to $5 \times 10^{-3}$ losses per cell division (Lackner et al., 1994; Hedgecock and Herman, 1995; Miller et al., 1996). As mentioned above, extrachromosomal elements, especially arrays, can also suffer sequential losses, such that positive clones are established after embryogenesis is well underway. 
We now come to the focus of this section: an examination of the mosaics with the compound microscope for the true nature of their losses. A record of the positive and negative nuclei of each mosaic can be made and the point of loss of the extrachromosomal element determined. The mosaics are mounted onto slides for examination with the compound microscope. Prepare a slide having a flat pad of 5\% agar or Difco noble agar as described (see Methods in cell biology). Place a drop of 3 to $5 \mu \mathrm{l}$ of M9 buffer on the surface of the pad, transfer the candidates with your normal pick into the drop, and then gently position a glass cover over the drop. Examinations may be more convenient if the worms have been immobilized by means of an agent added to the drop of M9 buffer, to the agar pad, or to both. Two possibilities are 5 to $10 \mathrm{mM}$ sodium azide and $1 \mathrm{mM}$ levamisole. It is often necessary to determine empirically an optimal concentration of the anesthetics such that paralysis is achieved without detrimental effects. Sodium azide can, for instance, destroy green fluorescence if the initial signal is weak. Although SUR-5::GFP can be examined in the presence of sodium azide, investigators not yet adept at scoring may prefer to use levamisole. The ideal situation is to avoid anesthetics because of their effects on viability and morphology. Although worms can survive these anesthetics, it might be more advantageous to avoid them in cases where one hopes to rescue worms from the slides in order to ascertain the effects of mosaicism on subsequent growth, development, or fertility.

Although the nuclei of the intestine can be difficult to judge, most other cells can be examined for the Ncl phenotype in larvae. Some of the cells in which SUR-5::GFP is expressed (and their origin in the cell lineage) are as follows: from ABalap: CANL (L, Left), RID, CANR (R, Right), ALA, and RMED (D, Dorsal); from ABalpa: m3L and m3VL (VL, Ventral Left); from ABalpp: ASKL and ADLL; from ABarap: m3R and m3VR (VR, Ventral Right); from ABarpp: ALML, BDUL, ALMR, and BDUR; from ABplaa: ASIL; from ABplap: PLML, ALNL, HSNL, and the vulva (or its precursor cells); from ABplpa: excretory gland L, excretory cell, and rectal epithelium D; from ABplpp: hyp8/9, rectal epithelium VL, and anal depressor muscle; from ABpraa: ASKR, ADLR, and ASIR; from ABprap: PLMR, ALNR, HSNR, and the vulva (or its precursor cells); from ABprpa: excretory gland R; from ABprpp: hyp8/9, rectal epithelium VR, DVA, and body muscle; from MSaa: m7D, m8, and body muscles; from MSap: left coelomocytes, posterior distal tip cell, dorsal coelomocytes, and those body muscles not from M; from MSpa: m6D and body muscles; from MSpp: right coelomocytes, anterior distal tip cell, and body muscles; from E: all intestinal cells; from Ca: DVC and body muscles; from Cp: hyp11 and body muscles; from D: body muscles. Although perhaps a meaningless list for now, we shall point out many of these cells in the appropriate sections as we go through the animal, and you shall soon find yourself a budding expert on the anatomy. Later we hope to add more cells to the tutorial. We are open to suggestions, and we would be grateful if errors are brought to our attention.

\section{The tutorial}

\subsection{The tail}

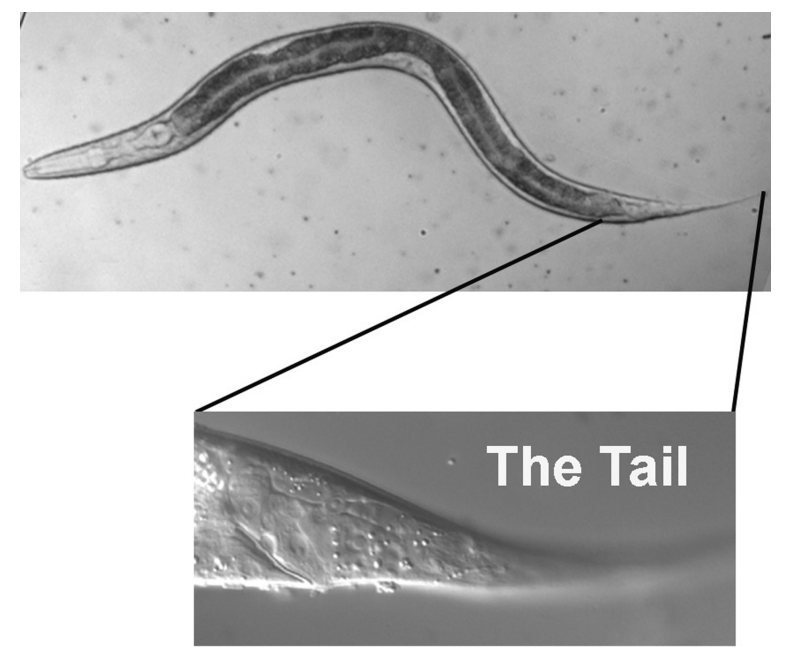

Figure 6. 


\subsubsection{Left lateral sections}

The tail, with its many cell types, presents a challenging beginning for the tutorial, but once we are familiar with some of the potential landmarks, we are poised to cruise through the middle part of the body and then tackle some of the complexities of the head. Our standard procedure will be to start with the extreme left focal plane and end at the central focal plane. Because the right focal planes are basically mirror images of those on the left, these will be left for the student to study. In the case of the tail, we know when to stop our transit through the planes, because we were introduced to the central focal plane when we studied the Ncl phenotype in Figure 3 and Figure 4. In fact, we shall use the cells $\mathrm{B}$ and $\mathrm{F}$ as prime landmarks while we study the tail.

As we start above the left side of an L4 hermaphrodite, the first view is of the cuticle, the collagenous exoskeleton of the animal. As we move the focus an increment towards the central focal plane containing B and F, the first nuclei to be seen are hypodermal (Figure 7). This is no surprise, of course, because the hypodermis secretes the cuticle of the outer body (the pharynx, the rectum, the vulva, the excretory duct cell, and neuronal support cells called socket cells also secrete cuticle). In the Figure, three nuclei from hyp7, the large hypodermal syncytium, are indicated. These are the posterior-most lateral nuclei of hyp7. (The bright area just anterior to them is the out-of-focus intestine. In this region, it joins the rectum, whose out-of-focus image can just be discerned.) Posterior to the hyp7 nuclei is another large nucleus. It is also hypodermal, but is not part of hyp7. It is a lateral hypodermal cell, also called a seam cell. In hatchlings, this hypodermal cell is a blast cell called TL. During the fist larval stage (L1), TL divides. In the L4 animal shown here, the descendant TL.appa is the seam cell. (A dot, such as the one between "TL" and "appa," is used to indicate cells that are produced during postembryonic development; the cells that arise during embryonic development do not have a dot in their cell-lineage designations. Also, it should be mentioned that the "a" stands for anterior and "p" for posterior. The daughters of TL are TL.a and TL.p. Thus, the seam cell shown here is the great-granddaughter of the anterior daughter of the TL blast cell.). The bodies of the seam cells have a distinct shape, and one can often see this shape with Nomarski optics. The cells resemble an "eye" or a nucleus with lateral wings. In Figure 7, the dorsal boundary of the seam cell can be seen (black line), but the ventral border is not very visible.
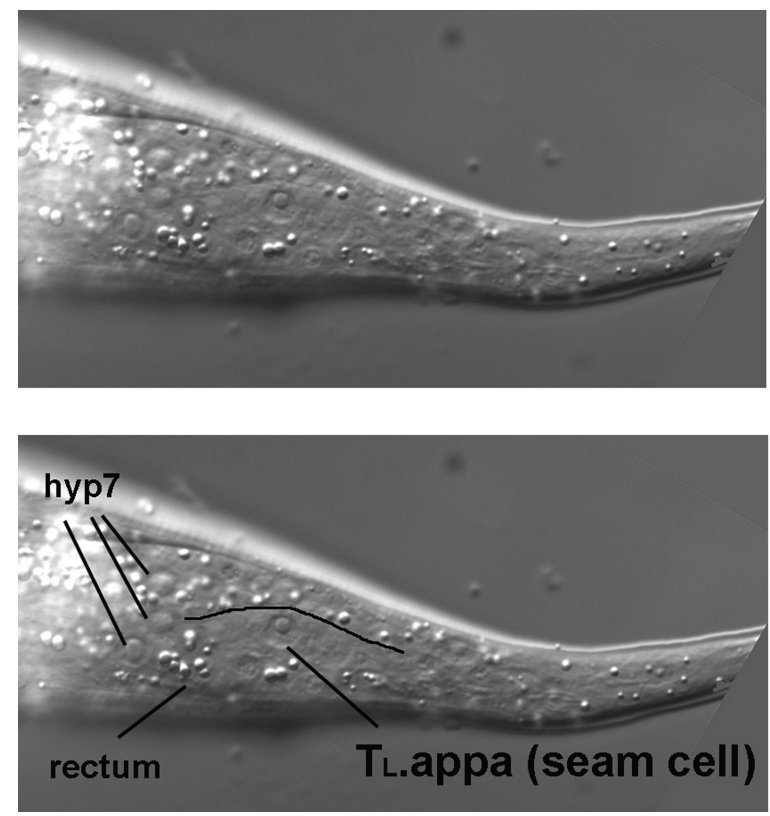

Figure 7. The extreme lateral nuclei of the tail.

The seam cell from TL is the posterior-most large nucleus in left lateral views. This is particularly evident when one examines the extreme lateral view of a hatchling (Figure 8). The seam cell (TL itself in this animal) is prominent, and the eye shape or "nucleus with wings" clearly visible. Because of tapering of the tail in this animal, the focal plane of TL is further towards the central plane; the hyp7 nuclei are left of it and not visible in this image. 

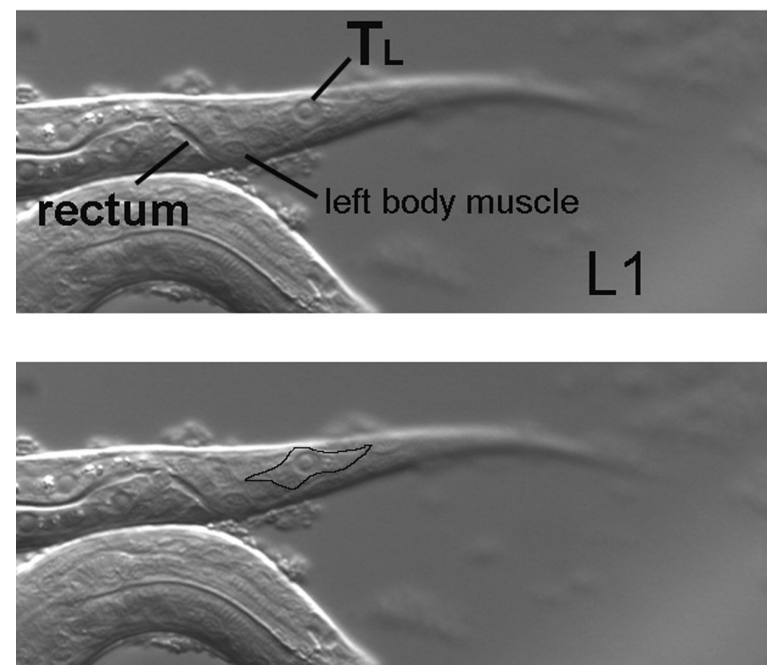

Figure 8. TL, the posterior-most seam cell on the left side of a hatchling.

Returning to the L4 larva, another incremental move of the focus brings the posterior-most ventral body muscle (Cappppv) on the left side into view (Figure 9). It is just posterior to the rectum. If we were to continue through the focal planes, we would encounter a body muscle (Cpppppv) in the corresponding position on the right side of the animal. These body muscles are therefore contralateral of each other. The two posterior-most body muscles on the dorsal side, in contrast, are not directly across from each other. Although one is in the left quadrant and other in the right quadrant, they are separated from each other along the anterior-posterior axis, as we shall see in due course.
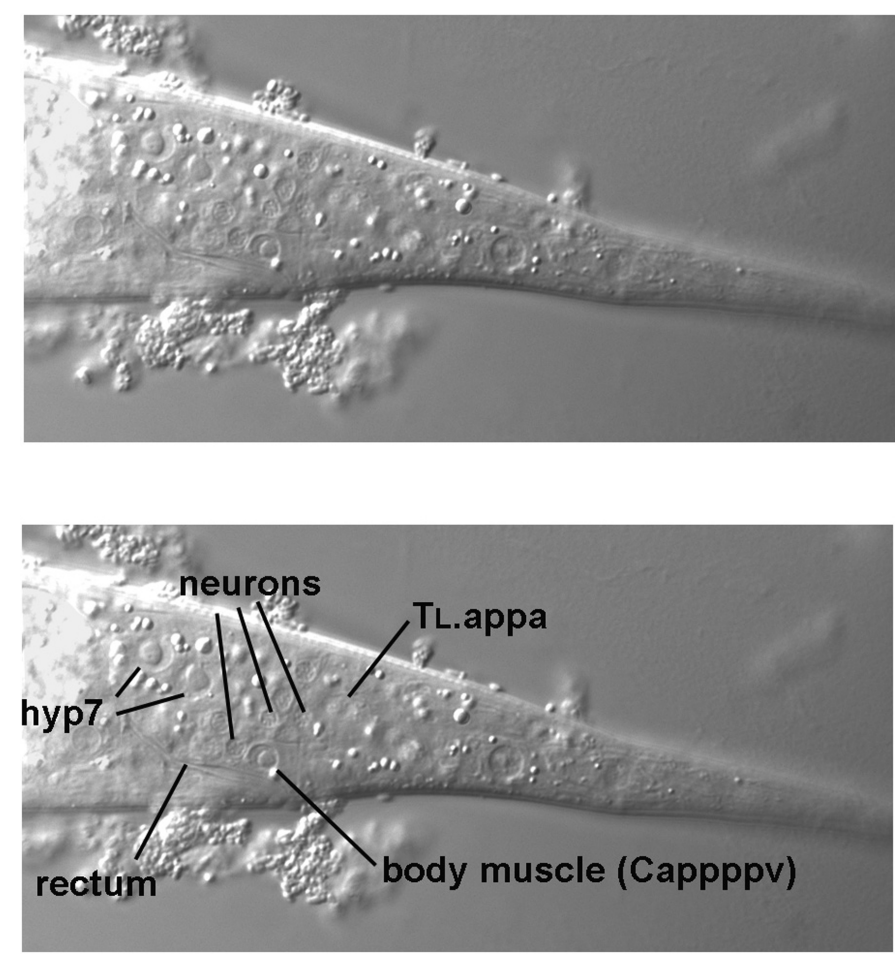

Figure 9. A ventral body muscle and cluster of neuronal cell bodies (the left lumbar ganglion).

Dorsal to the left ventral body muscle is a cluster of nuclei that are typical of neurons: the nuclei are small, "granular," and lack obvious nucleoli (for simplicity, only three are indicated in the figure, but others are clearly evident nearby). These are the nuclei of the left lumbar ganglion. There is a corresponding ganglion on the right 
side. The nuclei on the left descend from $\mathrm{ABpl}$, and with one exception (PVR, which descends from $\mathrm{C}$ ), the nuclei on the right descend from ABpr. Thus, if using SUR-5::GFP as a marker, an ABpl- ABpr+ mosaic will have no green neuronal nuclei in this region on the left side. On the other hand, the neuronal nuclei on the right side will all be green.

Moving the focus a bit more (we can tell that we have not gone far, because, even though they are largely out of the focal plane, the Cappppv body muscle and some of the nuclei of the left lumbar ganglion can still be seen, but the posterior seam cell and lateral nuclei of hyp7 are not to be seen at all), we now see a nucleus near the dorsal edge that clearly appears to be neuronal (Figure 10). This is the cell body of ALNL. By moving the focus just right of the central plane, the contralateral nucleus of ALNR should come into view.
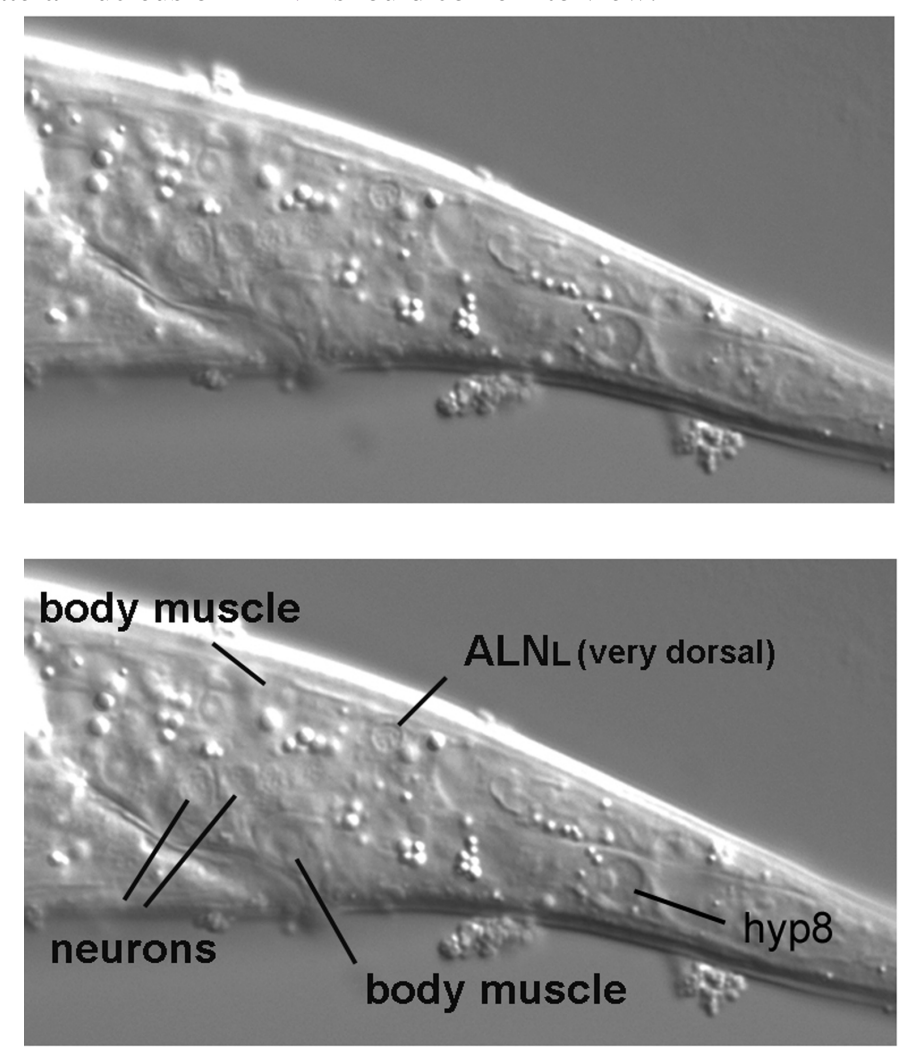

Figure 10. The cell body of the neuron ALNL.

\subsubsection{The central section}

By moving the focus another increment, we now revisit the central focal plane near the rectum (Figure 3). We are already familiar with the nuclei of the rectal cells B and F, which have distinctive morphologies. Just dorsal to $\mathrm{F}$ in these micrographs is the nucleus of K.a (K in hatchlings). A perhaps more typical view of K.a is present in Figure 11 , where K.a is slightly out of focus relative to F. This is not unexpected, because the nucleus of K.a is slightly left of the nucleus of F. If one goes slightly right of F, a nucleus similar to that of K.a comes into focus. This is the nucleus of $K^{\prime}$. The complete ring of rectum just dorsal to $\mathrm{F}$ is formed by K.a and $\mathrm{K}^{\prime}$, and their nuclei are near each other posterior to the lumen of the rectum. In contrast, the nucleus of the partner of B is anterior to the rectum, as is the nucleus of the partner of $F$. 

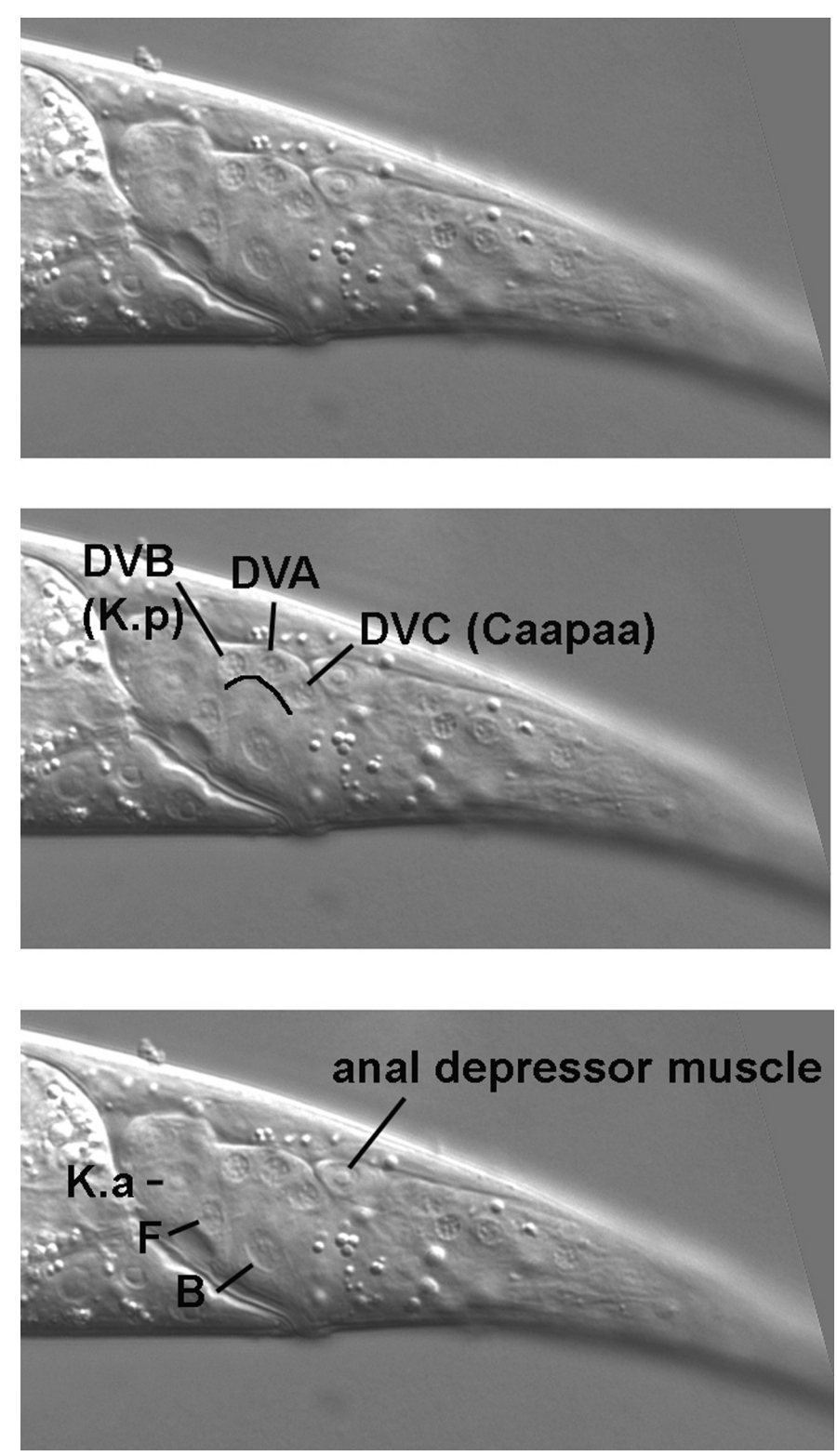

Figure 11. Several landmarks in the central focal plane of the tail.

The rectal cell B is worth a diversion. In males, B is a blast cell that produces many descendants, and it can be used to distinguish males from hermaphrodites in the first larval stage. Although the B cells of the sexes are indistinguishable at hatching, the B cell of a male becomes much more prominent during the L1 stage (Figure 12). Later in L1, the distinction becomes greater, for B will divide in the male, and two large nuclei, the daughters, will now be prominent in this region. The distinction between the sexes becomes increasingly dramatic with Nomarski optics after this stage. 

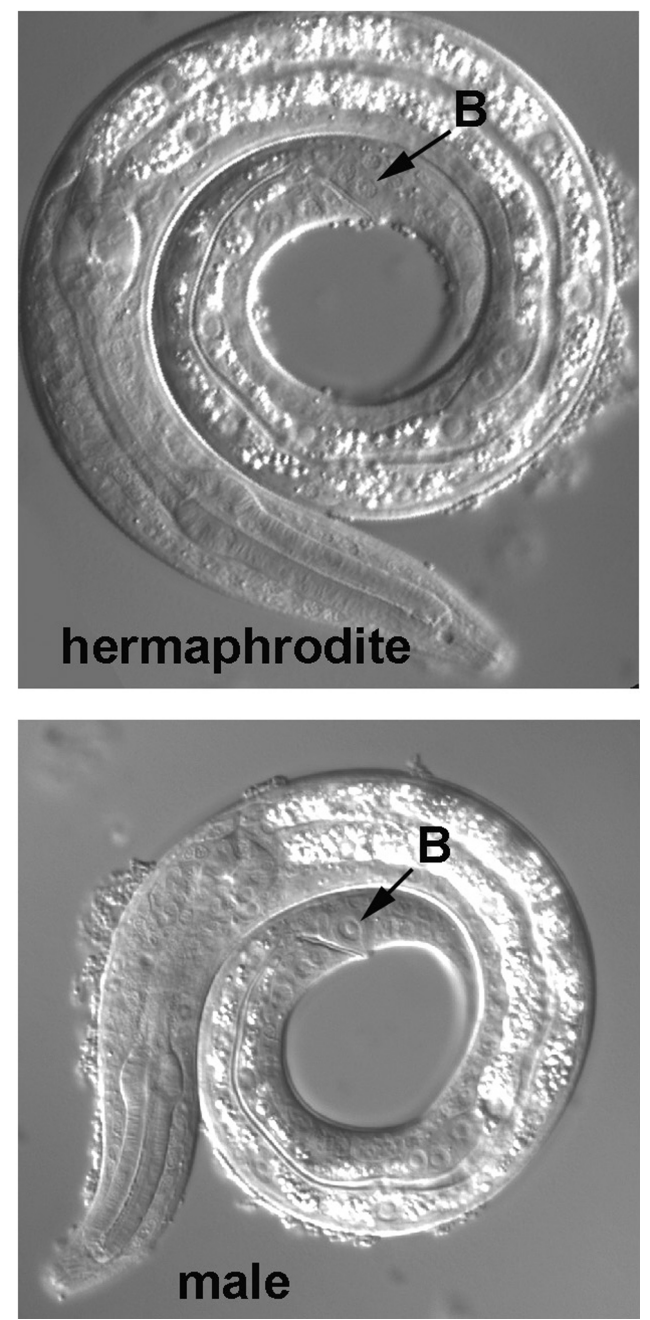

Figure 12. The rectal cell B, prior to its division in an L1 male, with the corresponding image from an L1 hermaphrodite.

Returning to the central section of the tail of hermaphrodites, another landmark is a distinct set, often in the form of an arc, of three neurons just posterior to the junction of K.a and K' (Figure 11). DVB, the anterior nucleus of this set, is the sister of the rectal cell K.a discussed above. Neither of these cells is present in hatchlings. During the first larval stage, the rectal cell K divides. One daughter, K.a, supersedes its mother, and the other daughter, K.p, becomes the DVB neuron. From the point of view of the cell lineage at large, DVB descends from ABpl, and the middle nucleus of the arc, DVA, from ABpr. The cell lineage of DVC, the posterior nucleus, on the other hand, is dramatically different. It is one of the two neurons that descend from the blastomere $\mathrm{C}$. Thus, in a mosaic in which only $\mathrm{C}$ has produced a positive clone with the marker SUR-5::GFP, DVC will be one of only two neuronal nuclei in the tail that will fluoresce green. The other neuron is PVR (Caappv), which was mentioned above. Its nucleus lies to the right of its close relative DVC.

Also obvious in the central section is the nucleus of the anal depressor muscle (Figure 11, lower panel). It can be easily distinguished from the posterior-most body muscle (Cappppd) on the dorsal side, because they are clearly separated by the pseudocoelom; the anal depressor is clearly internal to the dorsal body muscle (Figure 13). Also, the body muscle, being in the left dorsal quadrant, is usually to the left of the nucleus of the anal depressor. In the inset in the Figure, which is from a different animal, the body muscle was brought into focus by moving to the left of the central focal plane. The posterior-most body muscle from the right dorsal quadrant (Cpppppd) can be found on the right in the region where the intestine starts to taper on its way to join the rectum (Figure 14). Note that the right ventral body muscle and several nuclei of the right lumbar ganglion are also in this view. 

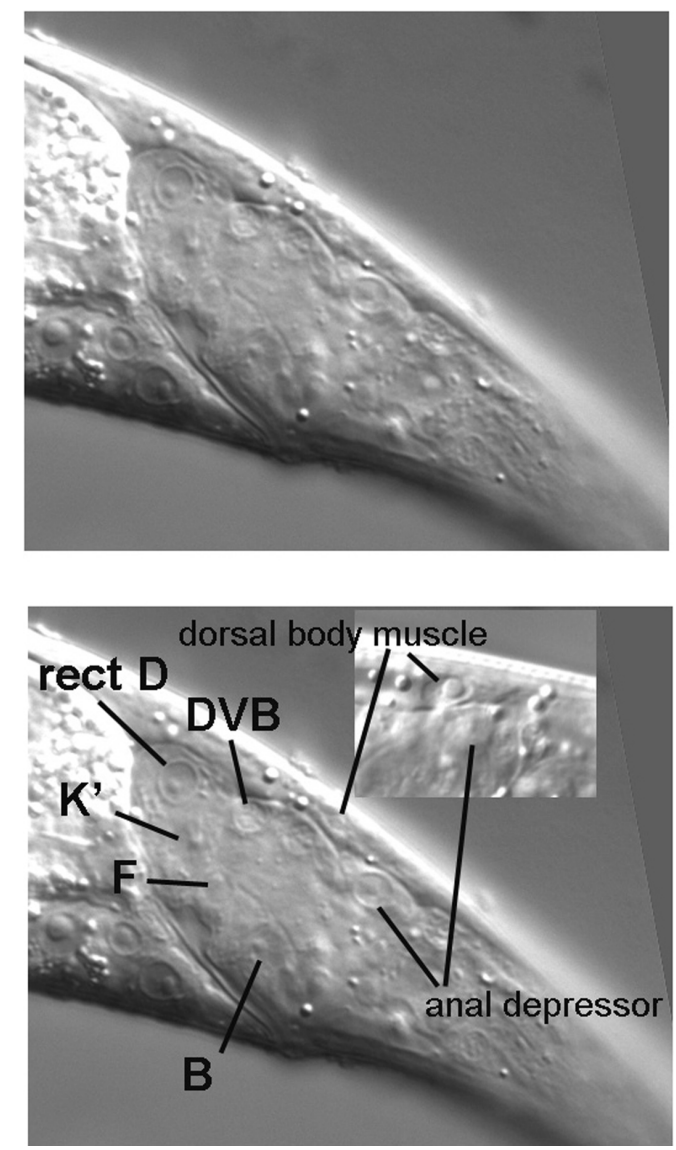

Figure 13. The nucleus of rect $D$ and the distinction of the left dorsal body muscle from the anal depressor muscle. Note in this micrograph that we are seeing K' (which is not completely in the focal plane) rather than K.a. This was concluded from slight adjustments of the focus.
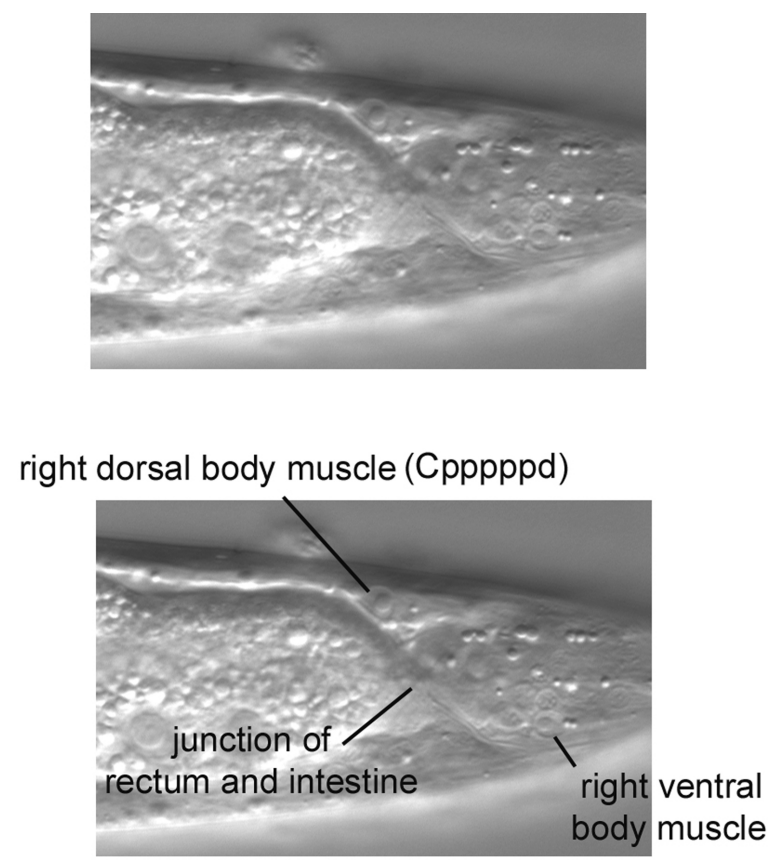

Figure 14. The posterior-most right dorsal body muscle. 
The nucleus of the rectal epithelial cell rect D is also present in Figure 13. It lies at the apex of the internal tissue of the tail, clearly separated from the body wall by the pseudocoelom. With a somewhat rounded shape, the morphology of the nucleus is distinct from those of B and F. Rect D is one of three bulb-shaped cells whose apical surfaces form part of a triangular ring at the junction of the intestine and rectum. We shall point out rect VL, another of these cells, when we study the region just anterior of the rectum. Serving as a reminder that we know less than we know, the function of the rect cells is not understood.

\subsubsection{Hypodermal nuclei}

The hypodermal nuclei of the tail are easy to see, and most are useful for mosaic analysis. Although most of the hyp7 nuclei are lateral (examples were seen in Figure 7 and Figure 9), a pair of hyp7 nuclei can be found in the central focal plane of the tail just posterior to the rectal cell B (Figure 3 and Figure 4). Posterior to the pair of hyp7 nuclei are three mononucleate cells, hyp8, hyp9, and hyp11, that form part of the tail hypodermis. They are easy to find, because their nuclei are large and form a triangle, with hyp11, which descends from the blastomere $\mathrm{C}$, at its apex (Figure 4). hyp8 and hyp9, the base of the triangle, are an example of lineal plasticity. They are defined not by their origins in the cell lineage but by their positions in the animal. The anterior cell is always hyp8, and the posterior always hyp9. With respect to the cell lineage, if hyp8 is ABplpppapap, then hyp9 is ABprpppapap, or vice versa. The posterior-most cell of the worm-the tip of the tail-is a binucleate cell, hyp10 (Figure 4). It is also requires caution during mosaic analysis, because one hyp10 nucleus is ABplppppppp, and the other is ABprppppppp. Thus, in a mosaic that suffered a loss of an array expressing SUR-5::GFP in ABp, both hyp10 nuclei would lack green fluorescence (or would both be Ncl, if $n c l-1$ is used as the mosaic marker). If, on the other hand, the loss is in ABpl but not in ABpr (or vice versa), both nuclei will fluoresce green or will be non-Ncl. The mRNA of the marker gene inherited by the ABprppppppp nucleus is translated in the cytoplasm of the binucleate cell, and the array-minus ABplppppppp nucleus therefore has access to the products of this translation in the shared cytoplasm. The same principle applies, of course, to a syncytium, such as hyp7.

\subsubsection{Exercises}

Now that we are familiar with some of the potential landmarks in the tail, we can use this knowledge and published diagrams to try as an exercise to identify other nuclei in the tail. If focused on the pair of hyp7 nuclei in the central focal plane, by moving the focus left, one can look for a neuronal nucleus PLML. It is often possible to discern the flattened cell body of this neuron. By focusing to the right of the hyp7 nuclei, the contralateral PLMR should come into view in roughly the corresponding position. Also in the tail are the left and right phasmids. The dendritic endings of the phasmid neurons are ciliated and breach the cuticle. They have support cells, called sheath and socket cells. An exercise for the student is to practice locating these nuclei based on the published charts.

\subsection{Just anterior to the rectum}

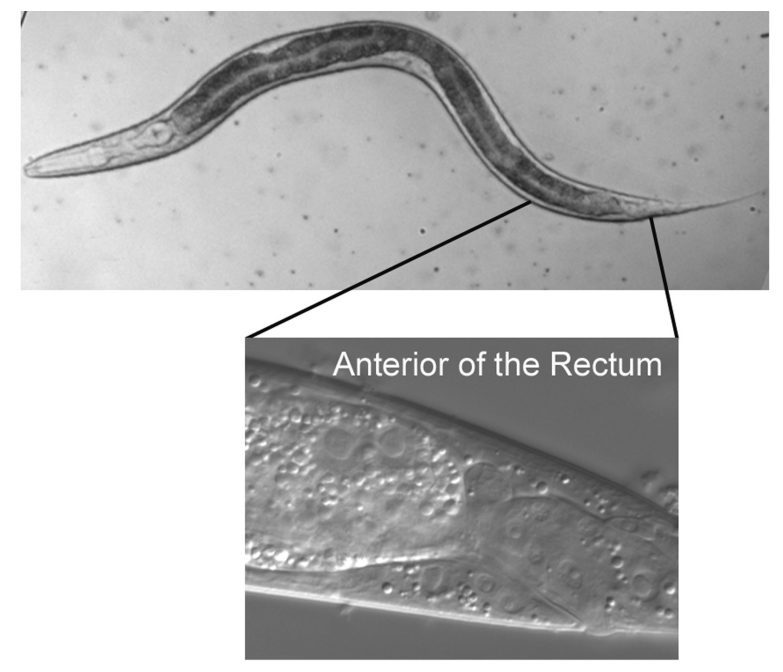

Figure 15. 


\subsubsection{Left lateral sections}

Our study of the region just anterior of the rectum will reinforce knowledge gained from the analysis of the tail. With the focus at the extreme left but right of the body cuticle, many of the lateral nuclei of hyp7 are visible (Figure 16). Also visible are several seam cells. Note, as we saw for the seam cell in the tail (which is visible at the posterior edge of the micrograph), the "eye" shape of these seam cells, which is enhanced by outlining for one of them.
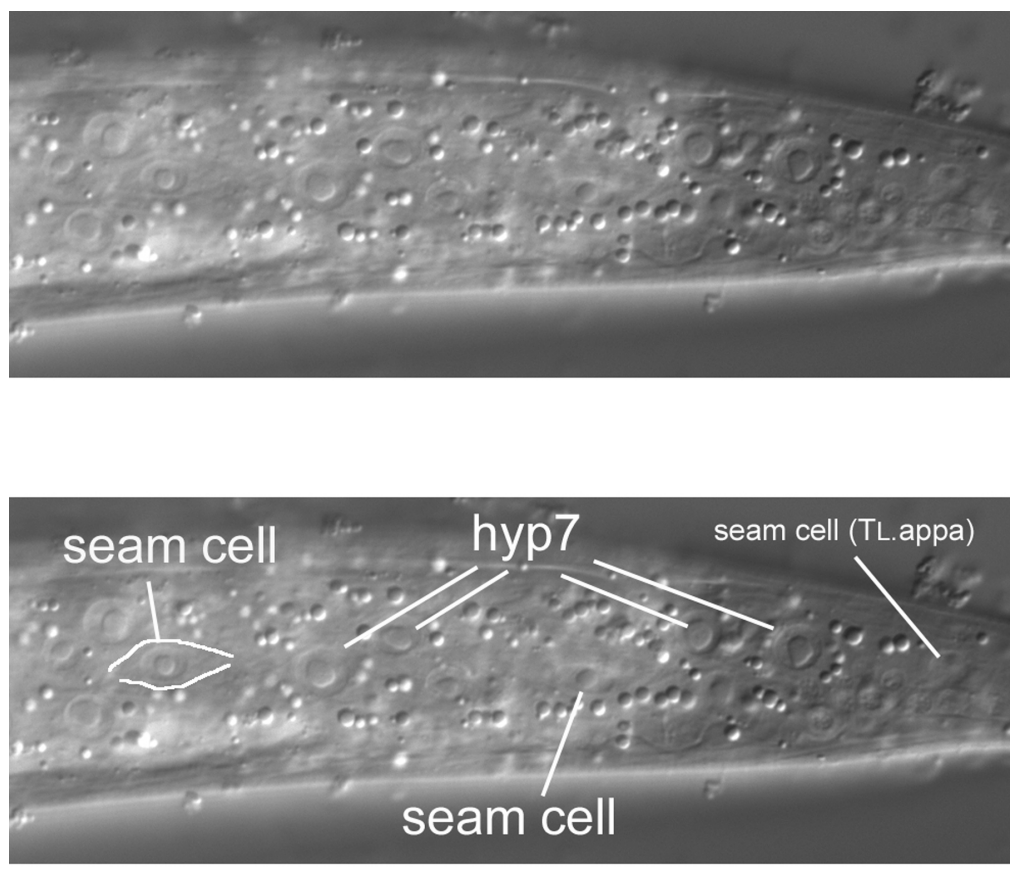

Figure 16. A far left view, with hypodermal nuclei evident.

Moving the focus towards the central focal plain (Figure 17), we encounter the characteristic nucleus and overall shape (partly outlined) of the rect VL cell. Note its resemblance to the rect D cell we saw in the tail (Figure 13). If we were to continue through the focal planes, we would see rect VR, the third member of this set. It is contralateral to rect VL. 

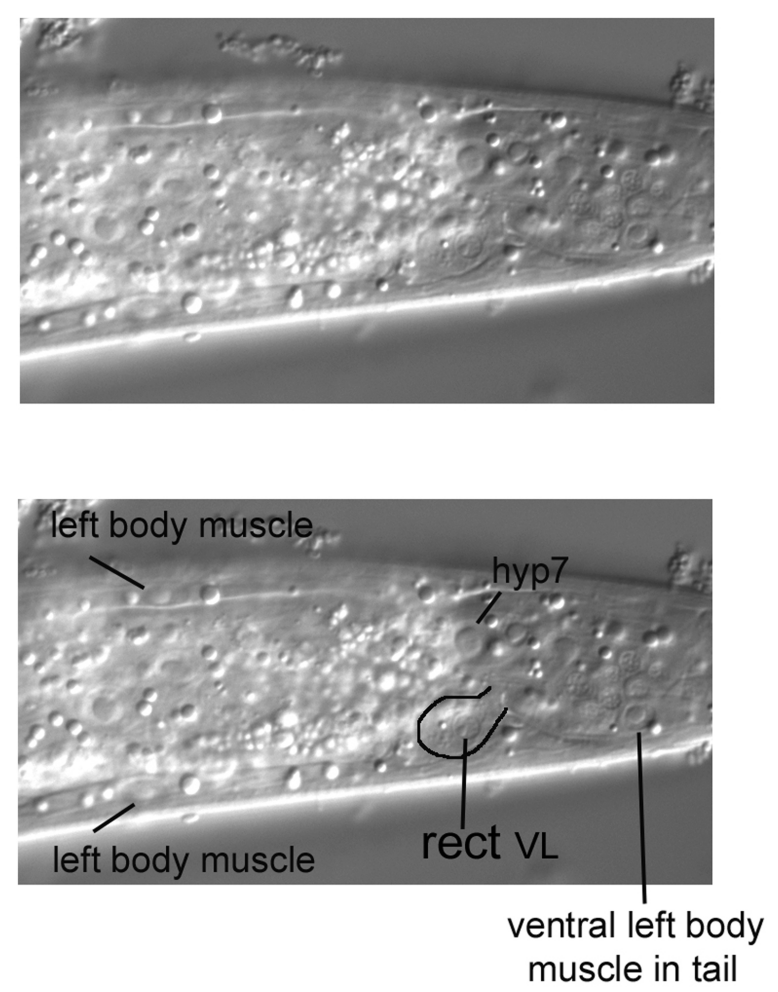

Figure 17. The characteristic nucleus and cell body of rect VL.

Also visible in Figure 17 are body muscles of the dorsal and ventral quadrants on the left side. The body muscles are useful for mosaic analysis, because they are mononucleate and descend from various embryonic blastomeres. Although caution is required, the body muscles of similar embryonic origin are to some degree in typical locations along the anterior-posterior axis of the animal. Useful for their identification, the nuclei of the body muscles have an ellipsoidal shape, and often the spindle shape of the muscles themselves can been seen.

\subsubsection{The central section}

The junction of the intestine with the rectum can be clearly seen in the central focal plane, which is familiar to us from our study of the tail (Figure 18). Note also the large nuclei of the intestine. Anterior to the rectum is P12.pa, the sole nucleus of hyp12, a small ventral hypodermal cell. The large nucleus further anterior is P11.p, which joined the hyp7 syncytium postembryonically. The Pn.p cells will be discussed in more detail in the section concerning the posterior part of the middle body. Between P11.p and P12.pa is the nucleus is of a rectal cell, either Y or U. We were not able to make a confident assessment. 

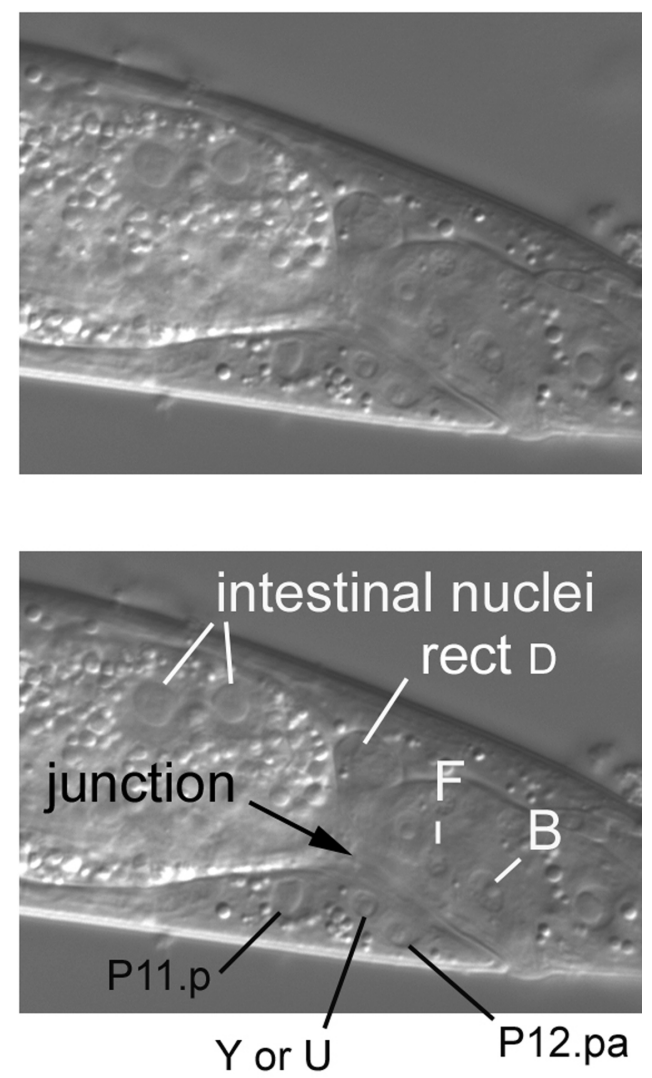

Figure 18. Centrally located nuclei immediately anterior of the rectum.

\subsection{The posterior part of the middle of the body}

The middle body is considered here to be the part that contains the intestine. Although long and somewhat monotonous relative to the head and tail, we shall nevertheless encounter interesting cells from various parts of the cell lineage. The four quadrants of body muscle are obvious in this region, and the gonad of either sex dramatically grows after hatching to take up a large volume of the region. In hermaphrodites, development of the vulva is one of the most intensely studied aspects of $C$. elegans biology. Extending the length of the middle body is the ventral nerve cord, which is easy to see in worms lying on their sides.

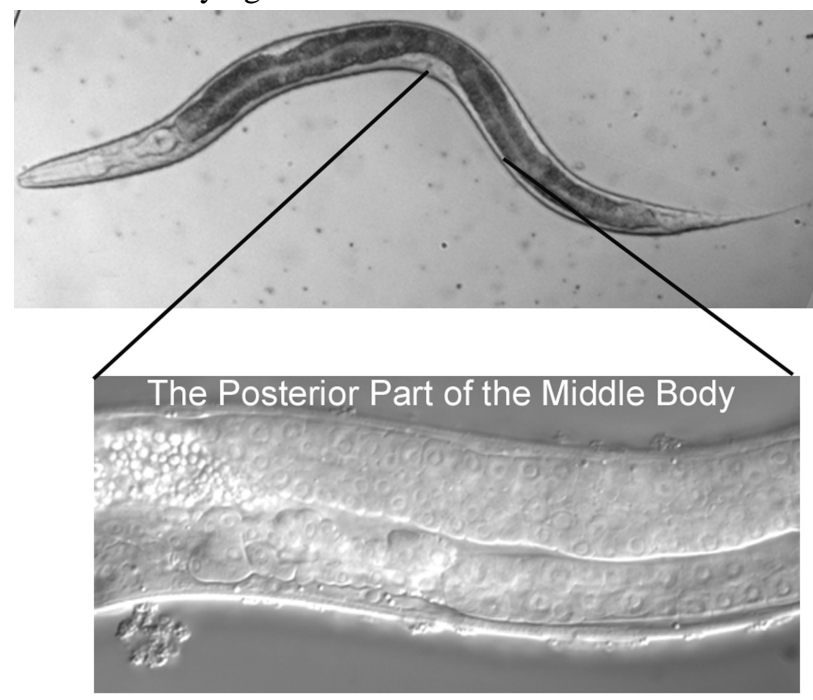

Figure 19. 


\subsubsection{Distinguishing Pn.p nuclei from body muscle}

It is important in the middle body to be able to distinguish the nuclei of the Pn.p "cells" from the nuclei of the ventral body muscles, which reside somewhat nearby. The problem can be acute in worms whose focal planes are not picture-perfect, because the size and shape of Pn.p nuclei resemble to some degree those of the body muscle. The Pn.p nuclei lie in the ventral cord in the central focal plane between the left and right quadrants of body muscle on the ventral side (Figure 20). The focal plane in the top panel is moderately left of the central focal plane. We know we are in the left half of the animal, because the posterior arm of the gonad is in focus in front of the intestine, as we saw in Figure 2. (The somatic gonad is still developing; the distal tip cell, which leads the migration of the arm, has ceased its posterior migration and is now migrating dorsally.) Note the elliptical shape of the nuclei of the body muscles. The spindle shape of the cell bodies of the dorsal muscle is evident here.
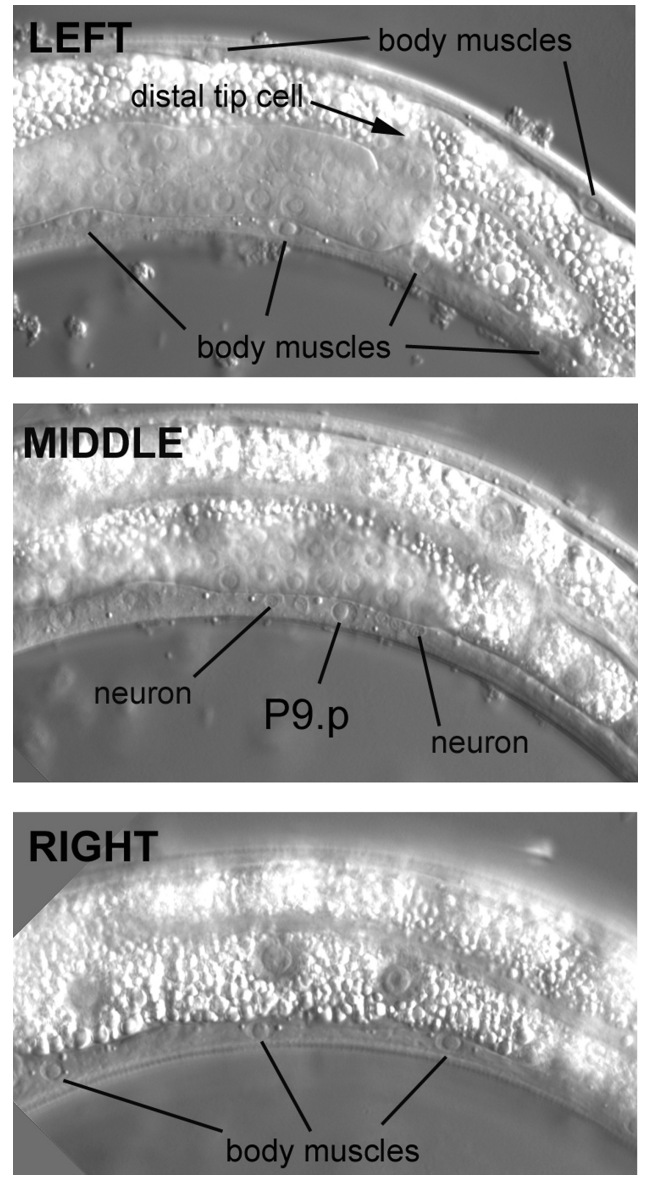

Figure 20. Careful adjustment of the focus helps to distinguish Pn.p nuclei from left and right body muscles.

Moving the focus an increment, the ventral cord of the central focal plane comes into view. Two types of nuclei are evident here. One type is about the size of a muscle nucleus. These are the Pn.p nuclei. They lie along the length of the cord among many examples of the second type, which are neuronal nuclei (only two are indicated in the middle panel). Shown here is the P9.p nucleus. Like the nuclei of the body muscles, the Pn.p nuclei have a prominent nucleolus. Moving the focus past the central section, we encounter the body muscles on the right side (note that the intestine is now in focus). Going through the focal planes in this manner-left (or right) body muscle, Pn.p and neurons, right (or left) body muscle-is a good routine to get into. It can greatly ease confusion, especially when the nuclei of the ventral cord do not fall precisely in the same focus along the length of an individual.

The P cells are dynamic during postembryonic development. The P9.p nucleus shown in the middle panel is actually part of the hyp7 syncytium, but it did not start out that way. At hatching, the $12 \mathrm{P}$ cells, the progenitors of the Pn.p nuclei, are not in the ventral cord. In fact, there are only 15 nuclei in the ventral cord, all of which are neurons. The $\mathrm{P}$ cells are situated instead on the flanks of the animal between and slightly ventral to the nuclei of the seam cells. There are six P cells on the left and six contralateral P cells on the right. (Now that we have experience 
with Nomarski images and the published diagrams, a fun exercise is to try to identify the lateral $\mathrm{P}$ cells in hatchlings.) Each contralateral pair is composed of left-right lineal homologues. During the L1 stage, the P cells enter the ventral cord, with the anterior-most contralateral pair forming the two anterior-most cells (P1 and P2) with respect to the length of the animal. The $\mathrm{P}$ cells divide soon after their invasion of the ventral cord. The anterior daughters ("Pn.a" cells) are neuroblasts that contribute most of the neuronal nuclei that populate the ventral cord after the late L1 stage (some of the Pn.a descendants undergo programmed cell death, which can be easily observed with Nomarski optics in older L1 larvae). The posterior daughters are the Pn.p cells. P1.p, P2.p, P9.p, P10.p, and P11.p fuse with hyp7 in the L1 stage. P5.p, P6.p, and P7.p, on the other hand, are the precursors of the vulva, as we shall see. P3.p, P4.p, and P8.p also have the potential to develop as vulval cells, but during normal development, they usually divide once during the L3 stage, and their daughters then fuse with hyp7. P12.p also divides; its anterior daughter, P12.pa, forms a small hypodermal cell, hyp12, just anterior of the rectum, as we saw in Figure 18.

The Pn.p nuclei require caution for mosaic analyses. As we saw for hyp8 and hyp9 in the tail, the P cells are defined by their positions along the anteroposterior axis and not by their strict cell lineage. The $\mathrm{P}$ cells come in left-right pairs with respect to the cell lineage, but the name of each member of the pair depends on which one ended up being anterior of the other after migrating into the ventral cord. There is an equal chance that the left $\mathrm{P}$ cell will end up anterior to or posterior of its contralateral right partner during the migration, and this chance is independent of the other pairs of $\mathrm{P}$ cells. P1 and P2 compose one pair, and P3 and P4 another, and so forth. If P1 is ABplapaapp, then P2 is ABprapaapp; if P1 is ABprapaapp, then P2 is ABplapaapp, and so forth for each pair. This plasticity is important to keep in mind when hoping for mosaics of the vulva equivalence group. For example, if P5.p is ABplappaapp, then P6.p is ABprappaapp, or vice versa. Furthermore, a P6.p with lineage ABprappaapp has half a chance in an individual of being next to a P7.p of lineage ABprappappp. Because both descend from ABpr, both will lack a wild-type copy of the gene under study in an ABpl+ ABpr- mosaic, but P5.p would have inherited the wild-type copy.

\subsubsection{Left lateral sections}

A view of the far left of an L4 larva reveals not only the expected hyp7 and seam-cell nuclei. Posterior of the developing vulva but anterior of the bend of the gonad is a cluster of six nuclei that are much smaller than the surrounding hypodermal nuclei (Figure 21). Although they can be difficult to find, they are the only small nuclei on the extreme left in the posterior part of the middle body. They can usually be located near the third seam cell posterior to the vulva. Four of the nuclei are of sensory neurons. One nucleus of the cluster is clearly larger than the other nuclei of the cluster, and it is the only one to have a noticeable nucleolus. This is the nucleus of the left postdeirid sheath cell, a support cell for some of the sensory neurons whose nuclei are in the cluster. Another support cell in the cluster is the postdeirid socket cell. The junction of the socket with hyp7 can be seen as a circle with the MH27 antibody or AJM-1::GFP construct (Francis and Waterston, 1991; Köppen et al., 2001). In hatchlings, there are no neuronal, socket cell, or sheath cell nuclei in this region. The sheath, socket, and two of the neurons descend postembryonically from the left V5 seam cell, while the other two neurons descend postembryonically from another blast cell, QL. (It is fun in hatchlings to try to find the left and right Q neuroblasts based on the published diagrams. Their nuclei can be distinguished from those of the $\mathrm{P}$ cells and hypodermis in this very general region.) Because of asymmetric migrations of $\mathrm{QL}$ and its right lineal homologue $\mathrm{QR}$, or their descendants, there are only two neuronal nuclei (plus the sheath and socket nuclei) in the cluster on the right side. One of the QL descendants (QL.ap) migrates into the left lumbar ganglion we encountered in the tail, but the other descendants remain in the region. In contrast, all of the QR descendants end up in the anterior part of the animal; two are on the right side between the pharynx and vulva, and the other lies to the right of the terminal bulb of the pharynx. 

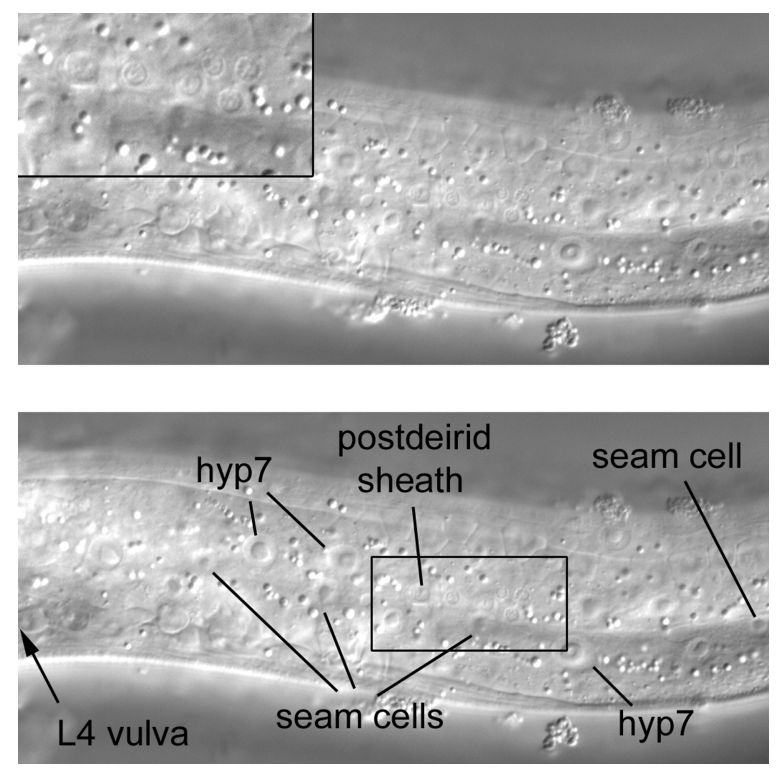

Figure 21. An isolated cluster of small nuclei (see inset) on the far left side of the posterior part of the middle body.

A short move of the focus brings the posterior arm of the gonad dramatically into view (Figure 22). The arm, led by the distal tip cell, has extended anteriorly almost the same distance as it did initially in the posterior direction before the distal tip cell turned dorsally (Figure 20). The posterior half of the left wall of the immature vulva can now be seen. A clear example of a body muscle is indicated posterior to the position of the vulva. Although, as stated above, the body muscles occupy general regions along the anteroposterior axis according to their cell lineage, this region of the body can be complicated after the first larval stage, when three left and three right dorsal muscles and four left and four right ventral muscles intercalate among the existing body muscles (see section 2.3.1). The existing body muscles arose and assumed their positions during embryogenesis (most in the region of interest descended from the blastomere $\mathrm{C}$ ). In contrast, the invading muscles derive postembryonically from a blast cell, $\mathrm{M}$ (MSapaapp), which will be discussed in more detail below. Another complication is ABprpppppaa, the sole body muscle that descends from AB. It is located just posterior to the vulva in the right ventral quadrant. If using SUR-5::GFP as a marker, in an AB+ P1- mosaic, this will be the only body muscle to fluoresce green. Seeing this is a source of simple pleasure. 

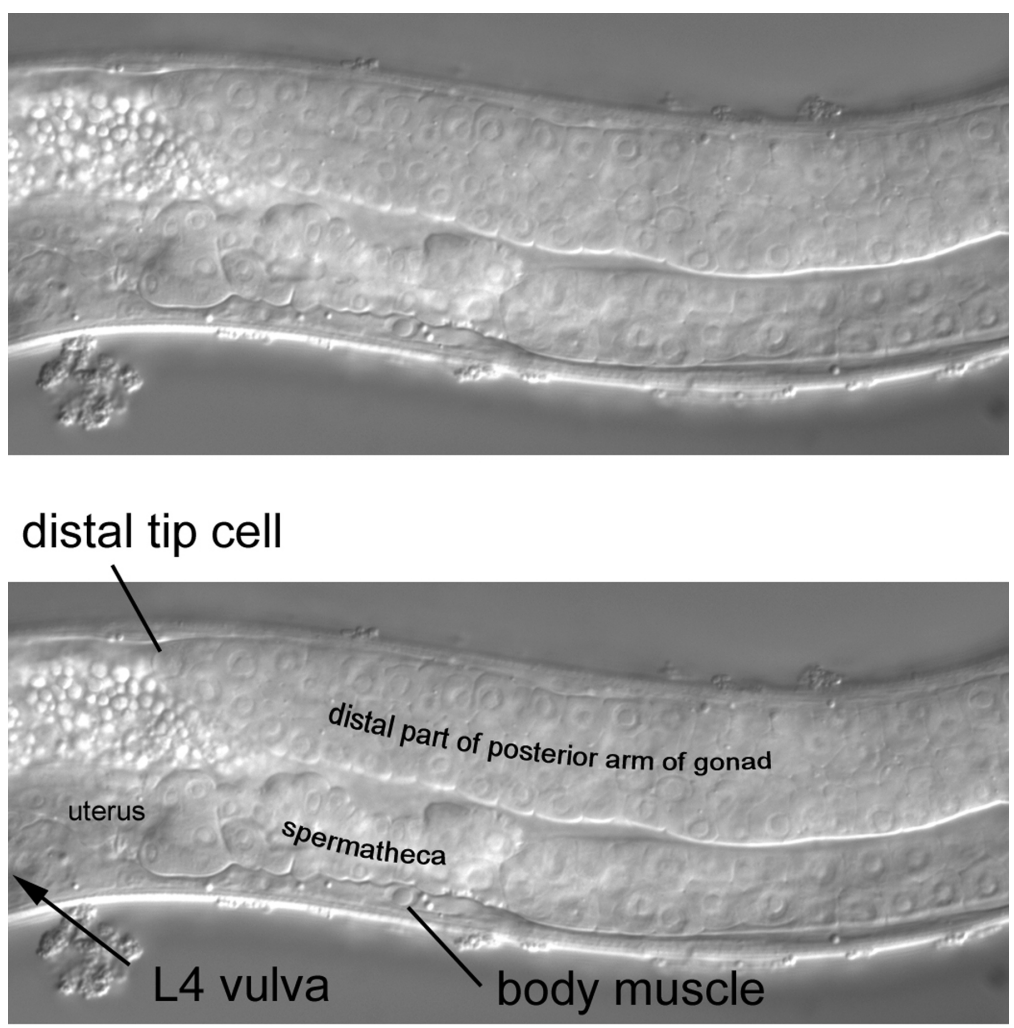

Figure 22. In a moderately left view, the posterior arm of an $\mathbf{L} 4$ gonad is prominent. Epithelization of the gonadal mesenchyme has produced a recognizable uterus and spermatheca

\subsubsection{The central section}

The posterior half of the L4 vulva can be clearly seen in Figure 23 (a more complete view will be presented when we discuss the middle part of the middle body). Two nuclei of the vulva can be seen just anterior to a large nucleus in the ventral cord. This nucleus is one of the daughters of P8.p (recall that in L3 larva, P8.p divides once). Both it and its sibling are now part of hyp7. Also in view are part of the lumen and part of the epithelial wall of the uterus. The uterus, like the other parts of the somatic gonad, descends from MS. To reinforce the lesson on distinguishing Pn.p nuclei from body muscle, we have moved the focus past the nuclei of the daughters of P8.p into the right side of the animal. Body muscles of the right quadrants are evident (Figure 24). 

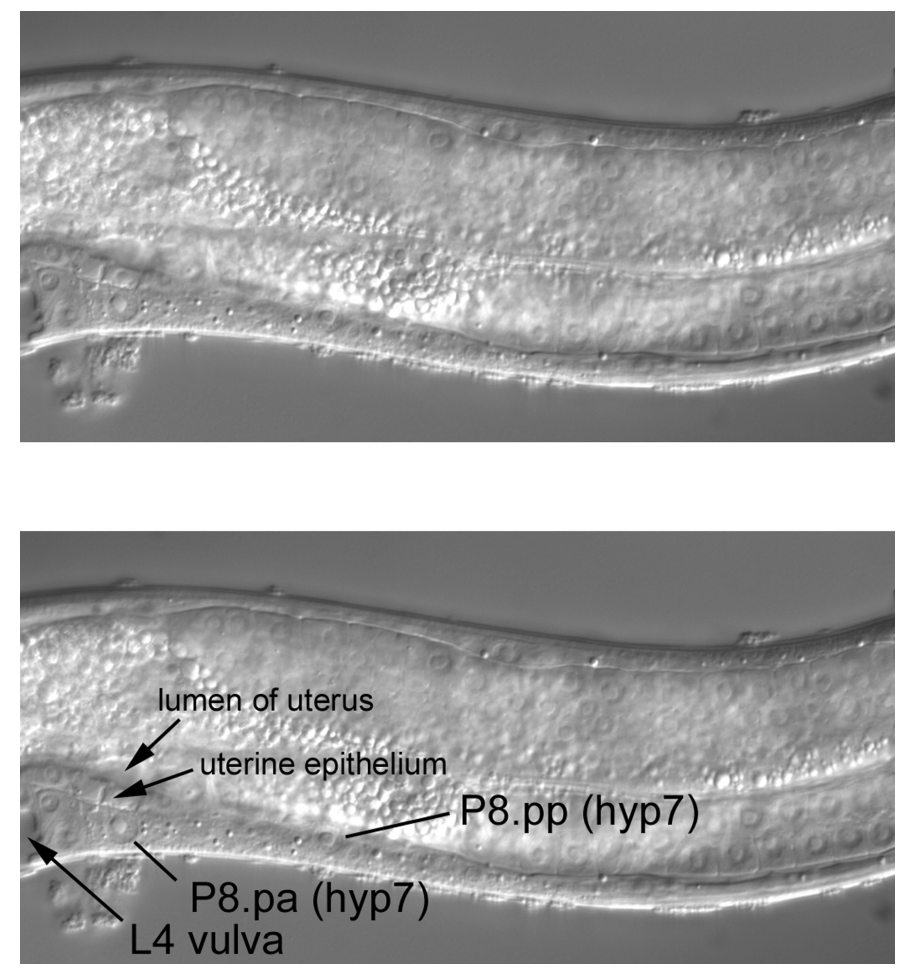

Figure 23. The central focal plane with two large nuclei in the ventral cord.
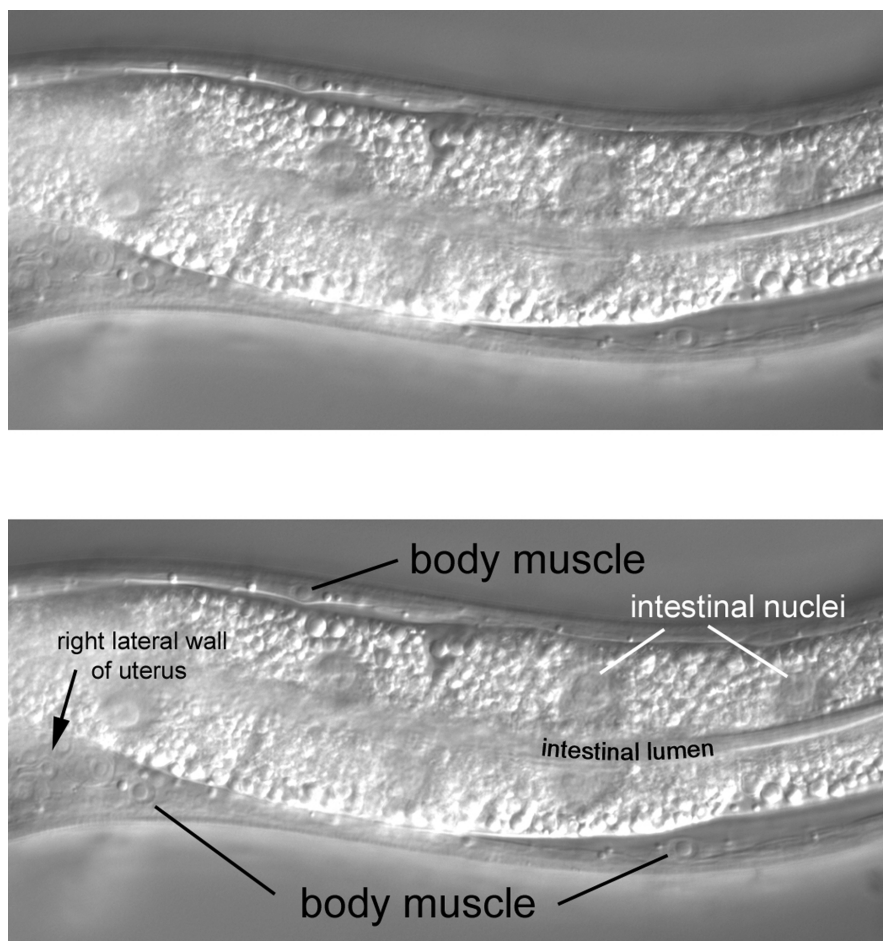

Figure 24. A view just right of the central focal plane.

Returning roughly to the central section, we shall search for two dorsal coelomocytes, which is not necessarily as easy as it sounds. A hermaphrodite is born with four coelomocytes, the two pairs in the ventral part that can be used to distinguish left from right. Two dorsal coelomocytes are added postembryonically from $\mathrm{M}$, the blast cell that 
is the progenitor of postembryonic body muscle, as we have seen above. Because it is small (and possibly obscured by intestine), a coelomocyte can be difficult to notice at first, but its distinct shape, the small size of its nucleus, and the presence of vacuoles help with unmistakable identification. Unlike the ventral coelomocytes, the dorsal ones are usually not immediately adjacent to each other. In Figure 25, one is somewhat anterior to the bend of the gonad. The other is near the bend and in a slightly left focal plane. This seems to be a typical location. If you re-examine the right panel of Figure 2, which is from a different animal, you can see a coelomocyte at the bend of the gonad.
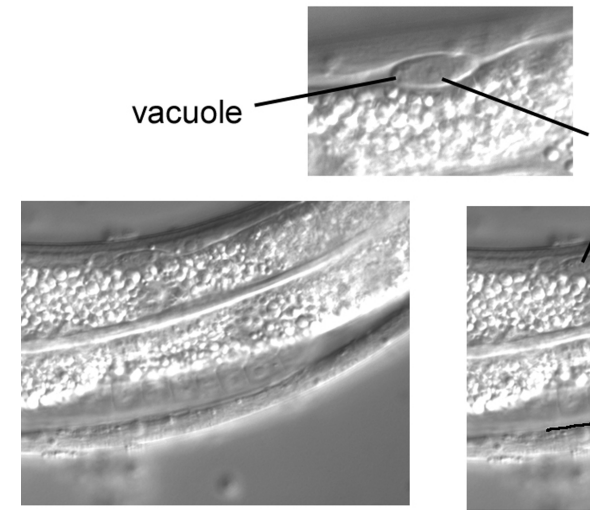
nucleus
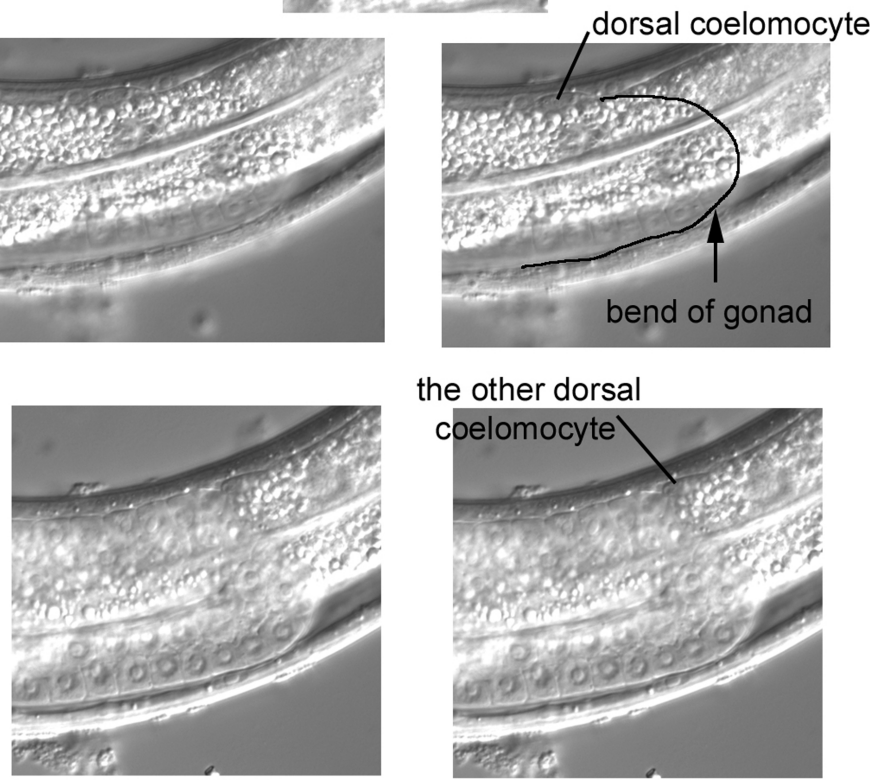

the other dorsal

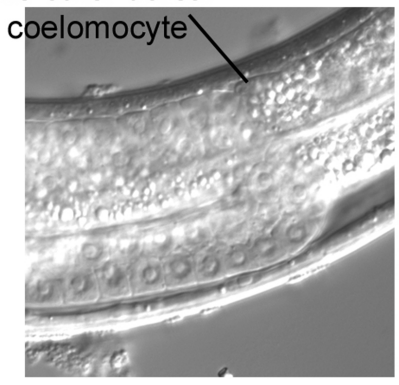

Figure 25. The sometimes elusive dorsal coelomocytes.

\subsection{The middle part of the middle body}

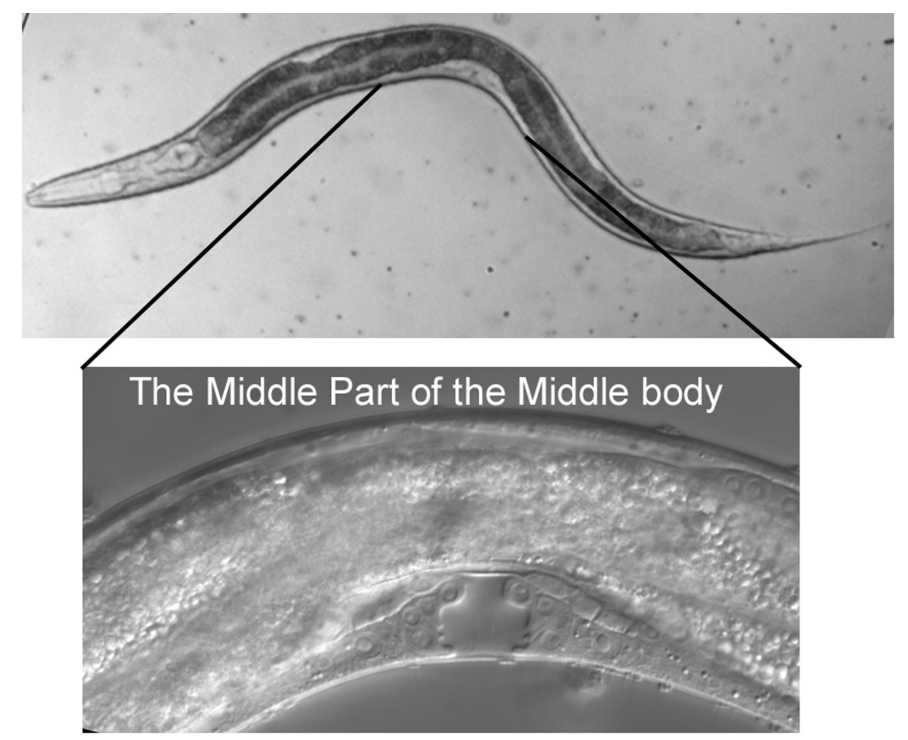

Figure 26. 


\subsubsection{Left Lateral Sections}

Hyp7 and seam-cell nuclei are of course visible in the nearly left-most focal plane (Figure 27). A neuronal nucleus can be seen left of the somatic gonad and slightly posterior to the anteroposterior midpoint of the gonad. This is the left hermaphrodite-specific neuron (HSN). The HSNR is contralateral. Although an HSN can be confused with neurons in the ventral cord in specimens that are not ideal for microscopy, the nucleus of the HSN is the only one in this area. It is distinctly lateral of and therefore dorsal to (one cannot be more ventral than the ventral cord) the neurons of the ventral cord. Note also that you can see the cell body flattened against the body wall and gonad.
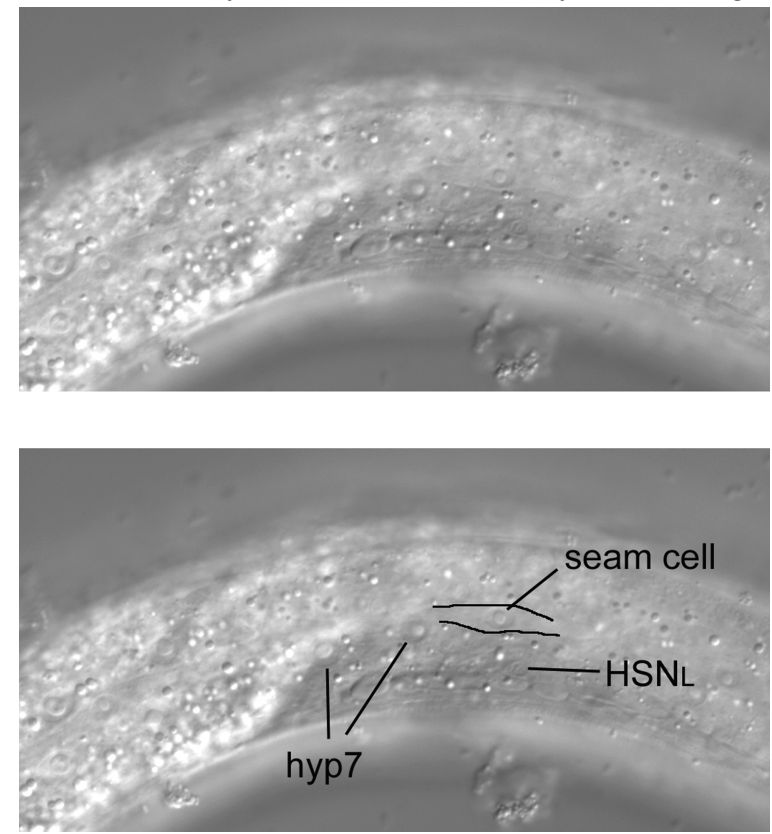

Figure 27. Far left, in the middle part of the middle body.

Although it presents a challenge, with a very short move of the focus towards the central section (note that the same seam cell is still visible), the nucleus of CANL can be seen (Figure 28). A small but noticeable nucleolus is always apparent, and the nucleus is clearly smaller than the surrounding hypodermal nuclei. It is dorsal of the HSNL nucleus and anterior of the anteroposterior midpoint of the gonad, and no other nucleus resembles it in the immediate region. More is said of the CAN cells below. 

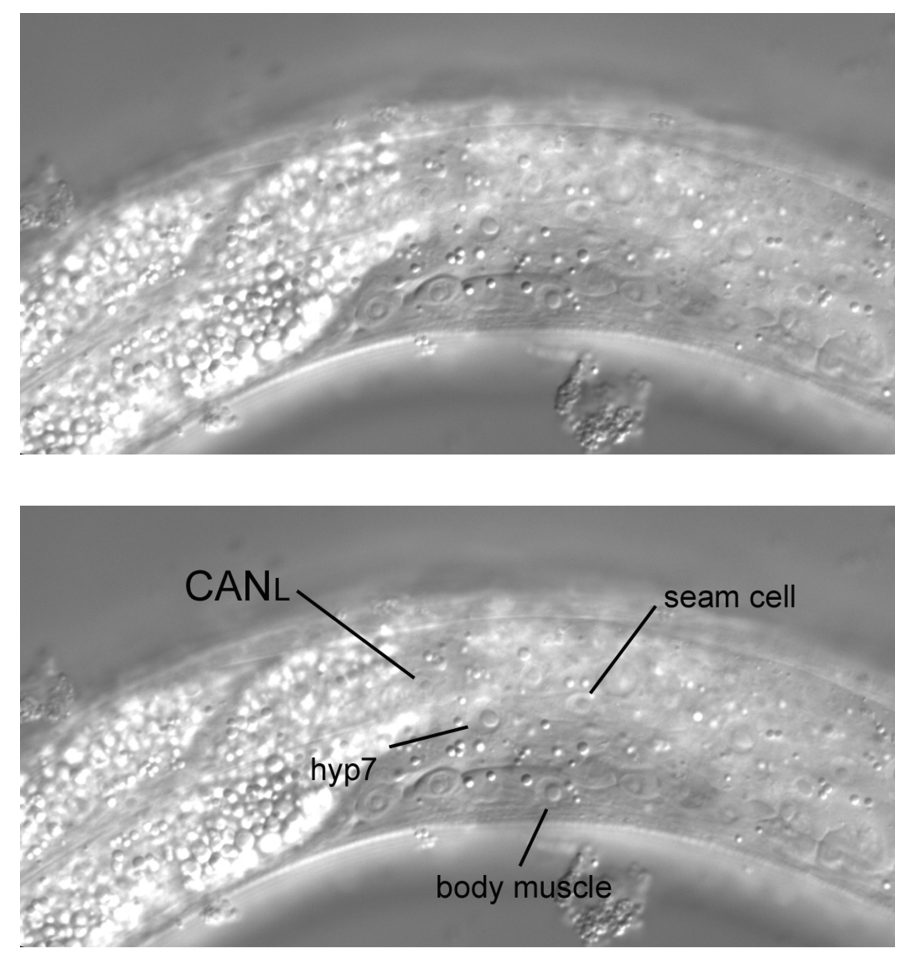

Figure 28. Not quite so far left, with the CANL nucleus visible. Note the change of the intestine from the left side to the right side of the animal. The position of the change is the anteroposterior midpoint of the gonad.

Moving the focus an increment, the vulva, the lumen of the posterior part of the uterus, and part of the posterior spermatheca of an advanced L4 larva are displayed (Figure 29). When L4 larvae are viewed with a dissecting microscope, the lumen of the uterus appears as a clear crescent, allowing unambiguous determination of this virginal stage at low magnification (cuticle separates the vulva from the outside until the molt to adulthood).
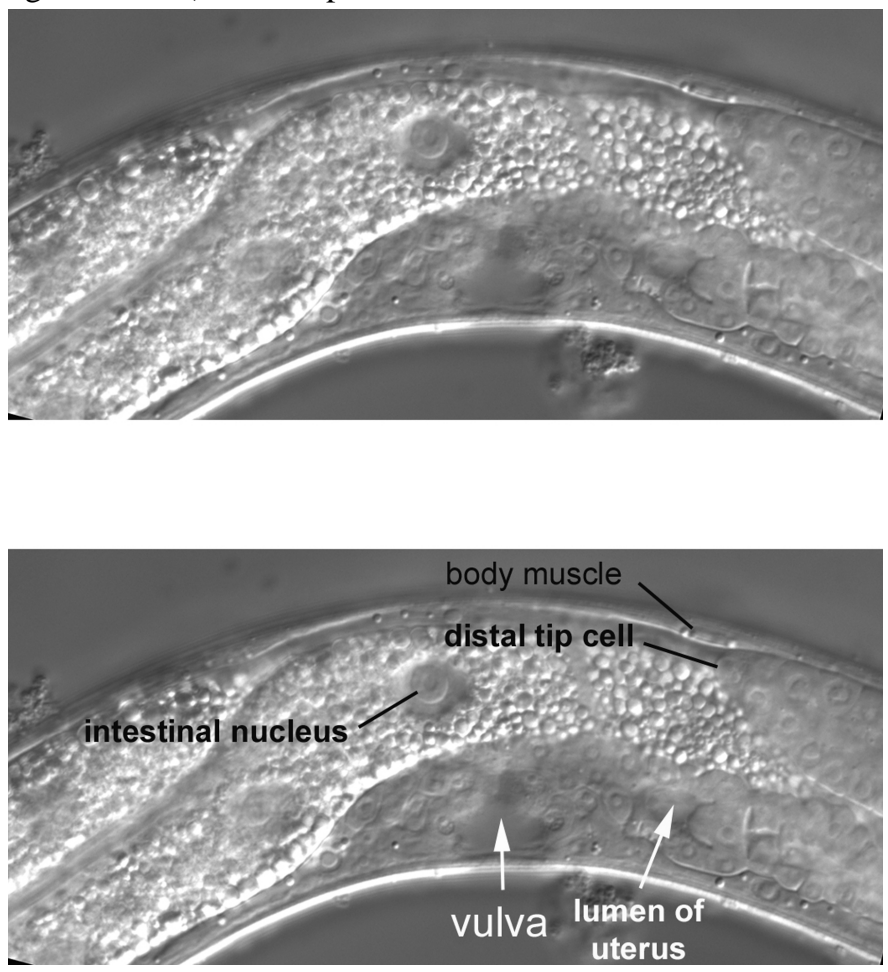

Figure 29. Still left of the central focal plane. 


\subsubsection{The central section}

A maximum longitudinal view of the late L4 vulva is presented (Figure 30). Several nuclei of the vulva can be seen. P3.p, P4.p, and P8.p, although they do not contribute to the vulva, are part of the vulval equivalence group [they have the potential to be specified as progenitors of the vulva (see Vulval development)]. During normal development, the cells usually divide once, and the daughters join the hyp7 syncytium. Although the cell lineage of the Pn.p cells was not analyzed in this animal, P4.pp and P8.pa are likely designations of the large ventral cord nuclei flanking the vulva. This assessment agreed with the expected number and positions of large nuclei along the ventral cord of this animal. Also of interest in this Figure is a full view along the anteroposterior axis of the lumen of the L4 uterus.
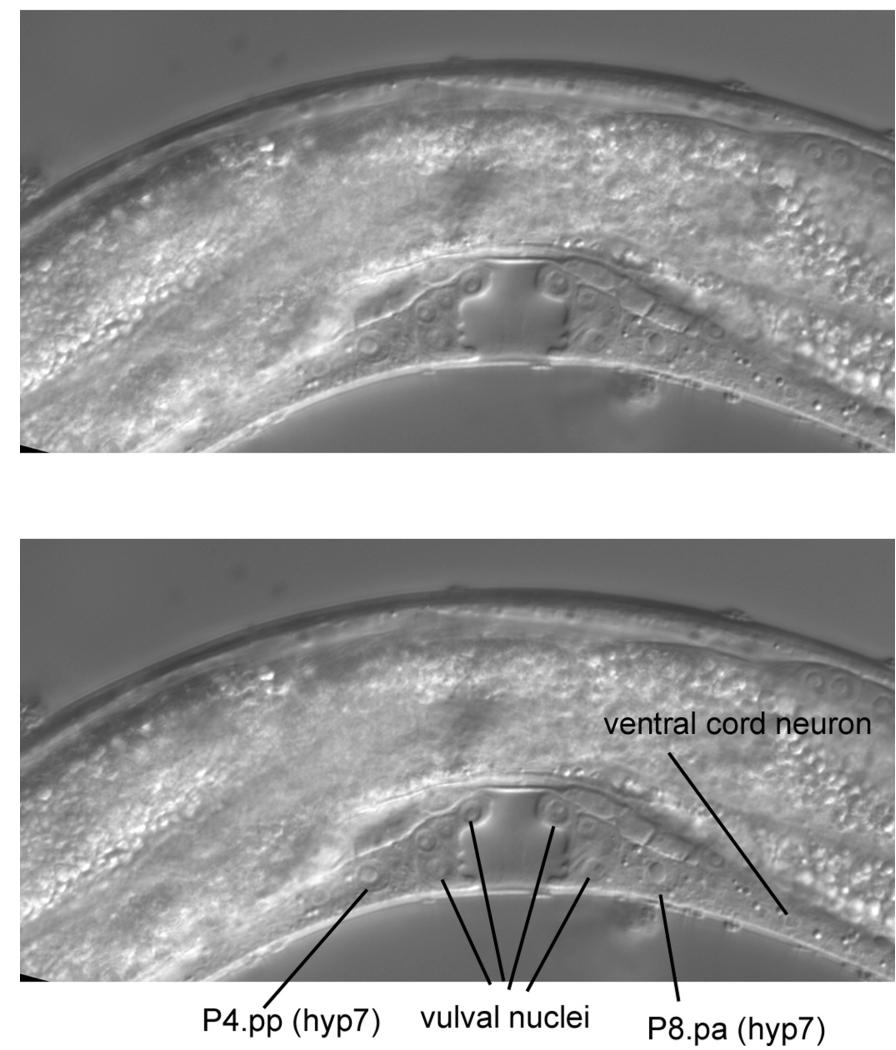

Figure 30. The central focal plane, with vulva and ventral cord.

\subsubsection{Additional views of the central section}

Although development of the gonad and vulva is too dynamic to be thoroughly described here (see Vulval development and Germline proliferation and its control), a few images of fleeting events in L3 hermaphrodites are presented here. The four images in Figure 31 concern the sex myoblasts and vulval precursor cells P5.p, P6.p, and P7.p. In the top pair of panels, P6.p can be seen ventral to the anchor cell, a distinct cell of the somatic gonad. A signal from the anchor cell to P6.p initiates development of the vulva. Moving the focus (next pair of panels) from the central plane to the left (notice the body muscle) reveals the consequence of another dynamic event that involves P6.p in an L3 larva at this time: born in the posterior part of the animal, the left sex myoblast has ceased its anterior migration after reaching a position lateral to P6.p. Precursors of the uterine and vulval muscles, the sex myoblasts descend from $\mathrm{M}$, the blast cell mentioned above as the precursor of postembryonic body muscles and coelomocytes. At the stage shown in this panel, the body of the sex myoblast can be distinguished (outlining) in front of (to the left of) the somatic gonad, although caution is required to avoid confusion of the nucleus of the sex myoblast with those of the somatic gonad. Moving the focus demonstrates that the left sex myoblast lies between the body wall and the left wall of the somatic gonad. [One way to become confident of seeing cells such as the sex myoblasts is to examine transgenic strains that express a cell-specific marker based on GFP. An even more sophisticated method has now been developed for C. elegans (Zhang et al., 2004).] 

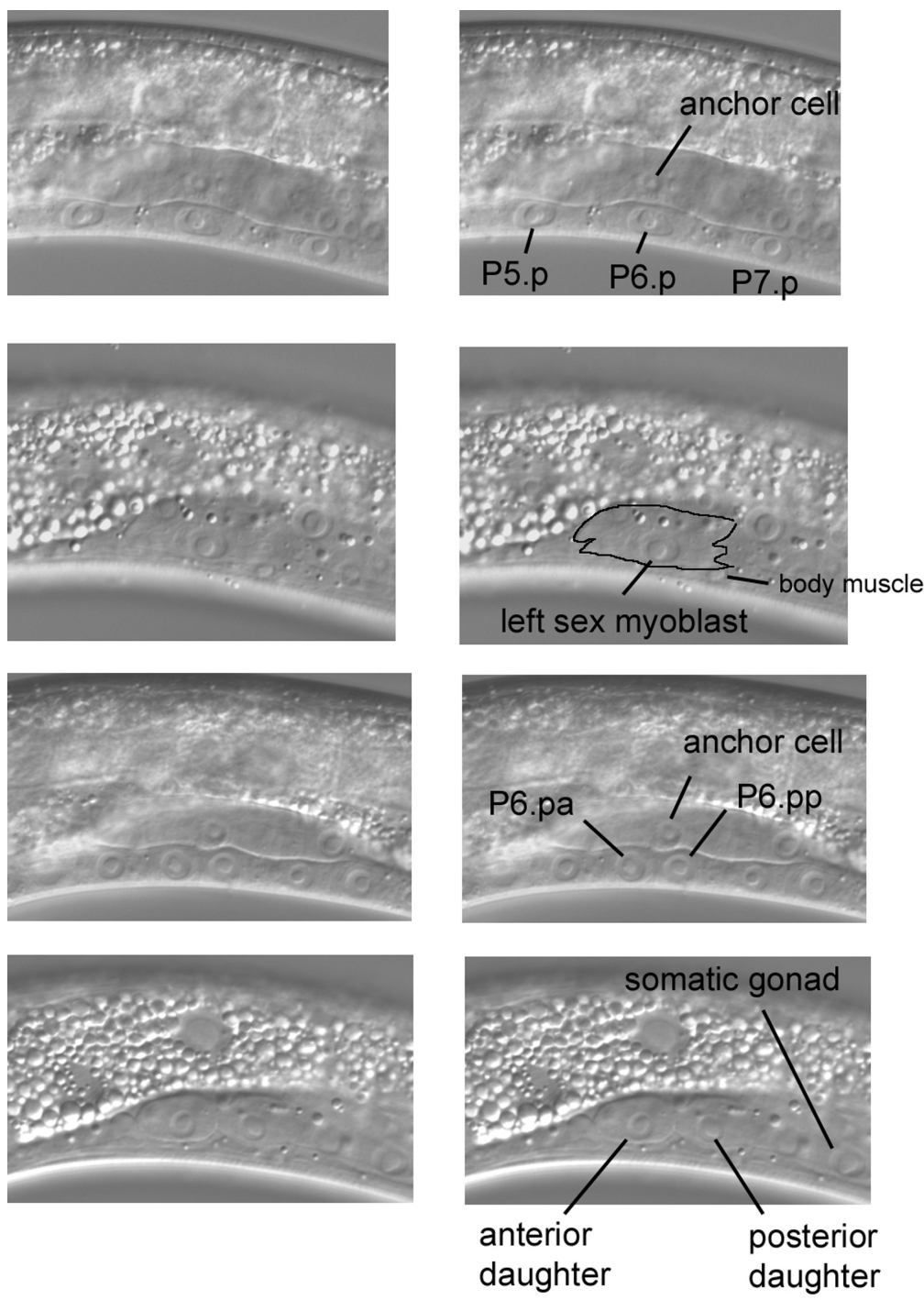

Figure 31. The dynamic Pn.p cells and sex myoblast of L3 larvae. Top pair of panels, central focal plane. Second pair, the sex myoblast left of the central plane in the same animal. Third pair, part of the somatic gonad and daughters of the vulval precursor cells P5.p, P6.p, and P7.p in the central plane of a slightly older L3 larva. Bottom pair, the daughters of the sex myoblast left of the central plane in the same animal.

The third and fourth images of Figure 31 are from an L3 larva slightly more advanced in development than the one in the first two images. Induced by the anchor cell, vulval development is now dramatically underway, for P5.p, P6.p, and P7.p have divided (third pair of panels). Note the positions of the daughters of P6.p relative to that of the anchor cell. If we move the focus to the left, we see that the sex myoblast has also divided (bottom panel). The bodies of the daughter cells clearly stand out in front of (to the left of) the somatic gonad (the posterior part of the gonad can be seen peeking out from behind the posterior daughter).

Because the CAN nuclei can be difficult to learn, a left lateral view from another L3 larva is shown in Figure 32. The CANL nucleus here (enhanced in inset) is clearly dorsal of and anterior of the anteroposterior midpoint of the gonad. The midpoint is easy to determine in this image, because the daughters of the left sex myoblast are easily seen, and the midpoint is between them. Other features are noteworthy in this image. The nucleus of HSNL can just be seen in front of (to the left of) the posterior daughter of the sex myoblast. Also visible is a ventral coelomocyte, one of the pair on the left side just anterior to the anteroposterior midpoint of the gonad. 

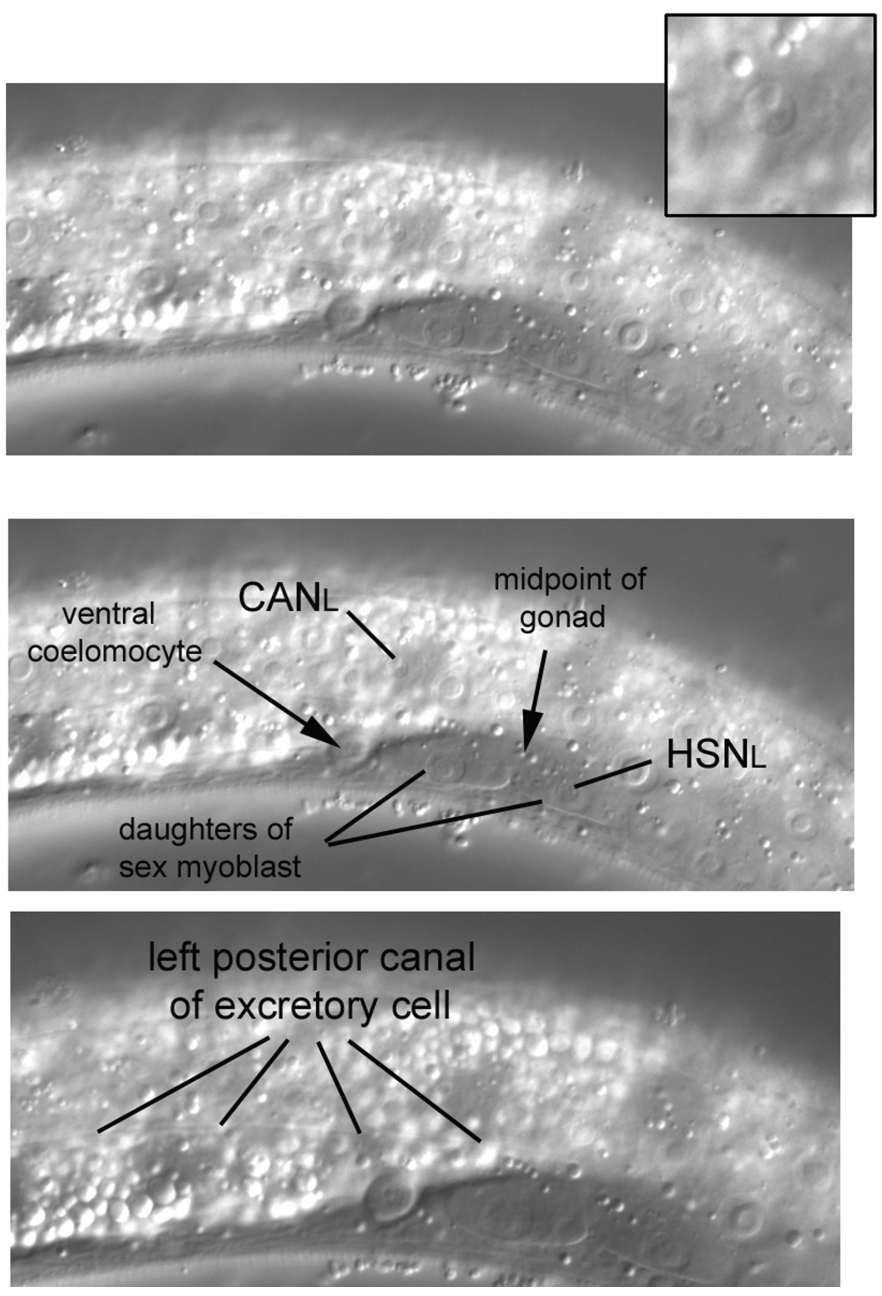

Figure 32. Far left in the middle part of the body. Another view of the CANL nucleus (enhanced in inset) and a glimpse of the left posterior canal of the excretory cell.

The left and right CAN cells are the posterior-most mononucleate cells that descend from $\mathrm{ABa}$. Of further note, the CAN cells have anterior and posterior processes that extend for much of the length of a worm. The processes are parallel to the canals of the excretory cell, whose nucleus we shall encounter in the posterior part of the head. The excretory canals (there are four in the shape of an $\mathrm{H}$ relative to a dorsal view of the long axis) and the processes of the CAN cells are sublateral to the hypodermal seam cells. The canals can sometimes be seen even in wild-type animals. By moving the focus slightly right in this N2 larva (note that the daughters of the sex myoblast are still mostly in focus), we were able to see the left posterior canal (bottom panel).

\subsection{The anterior part of the middle body}

This final section of that part of the body that contains the intestine will be brief. For practice, one can go through the drill of left body muscles-ventral cord with neurons and Pn.p nuclei-right body muscles. One can also look for the right pair of ventral coelomocytes. Because of the high refractility of the intestine, they are more easily seen in animals lying on their left sides. 


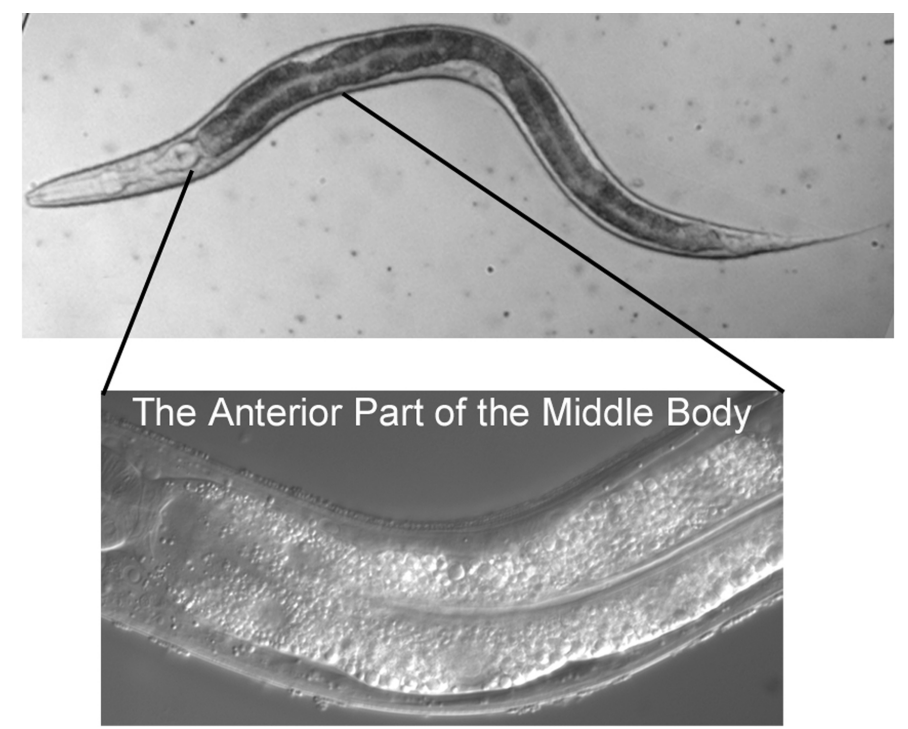

Figure 33.

\subsubsection{Left lateral sections}

The nuclei of two neurons, BDUL and ALML, can be seen in far left sections of this part of the animal. BDUL and its contralateral partner BDUR both descend from ABa. The nucleus of BDUL is extremely lateral in the region of the seam cells between the terminal bulb of the pharynx and the bend of the anterior arm of the gonad. Another means of finding the general region is to locate the midpoint of P1.p and P2.p, move to the far left plane, and then locate the seam cells. Although difficult to find at first (in large part because of the high refractility of the intestine), there are no other neuronal nuclei in this large region at the far left focal plane. Our first view of this region in an L3 larva contains hypodermal nuclei (Figure 34). Moving the focus ever so slightly towards the central focal plane brings the nucleus of BDUL into view (Figure 35). Transgenic lines that express GFP in neuronal nuclei (SUR-5::GFP will do) can be of much help when searching for BDUL: it will be the only small nucleus to fluoresce in this region.
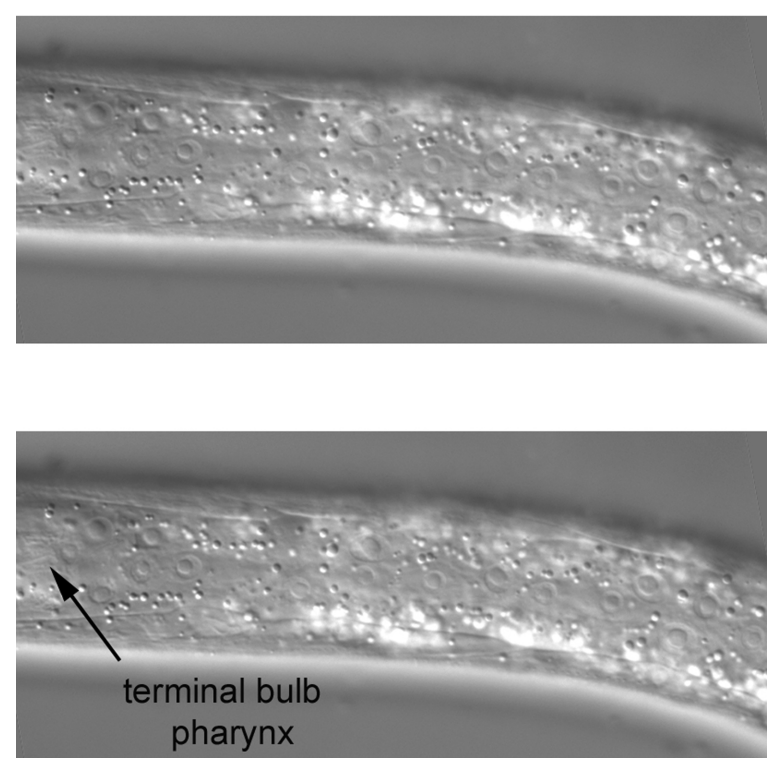

Figure 34. Hypodermal nuclei on the far left side of the anterior part of the middle body. 

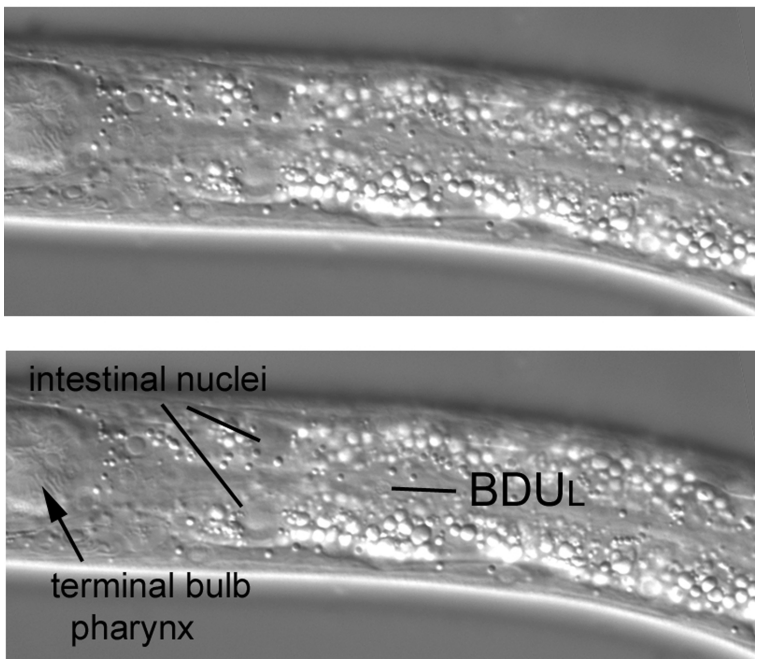

Figure 35. The isolated cell body of the neuron BDUL.

The right side of older larvae differs from the left in this region. There is a neuronal nucleus in addition to BDUR. This neuron, SDQR, descends from QR, the lineal homologue of QL that was mentioned when we studied the posterior part of the middle body. Intermediate descendants of QR migrate anteriorly from the natal position of $\mathrm{QR}$, and the nucleus of SDQR therefore is in an anterior region of the animal. In contrast, the cell body of SDQL, the lineal homologue of SDQR, is near the natal position of QL. In fact, the nucleus of SDQL is part of the lateral cluster that includes the nucleus of the left postdeirid sheath cell (Figure 21).

The second object of our search on the left side is ALML. It can be found more posteriorly than BDUL. It is also less lateral, appearing almost to be quite dorsal. Start near the anteroposterior midpoint of the gonad (the nucleus of CANL is a useful guide) and move anteriorly and dorsally a short distance (Figure 36). Although difficult to find, no other neuronal nucleus is in the general vicinity, and the cell body is often visible (Figure 36, inset). ALMR is contralateral. Another neuronal nucleus on the right flank is AVM. It resides near ALMR. There is no contralateral nucleus on the left side, because AVM, like SDQR, descends from the QR neuroblast. As was mentioned for SDQL above, the nucleus of PVM, the lineal homologue of AVM, is in the left lateral cluster seen in Figure 21. 

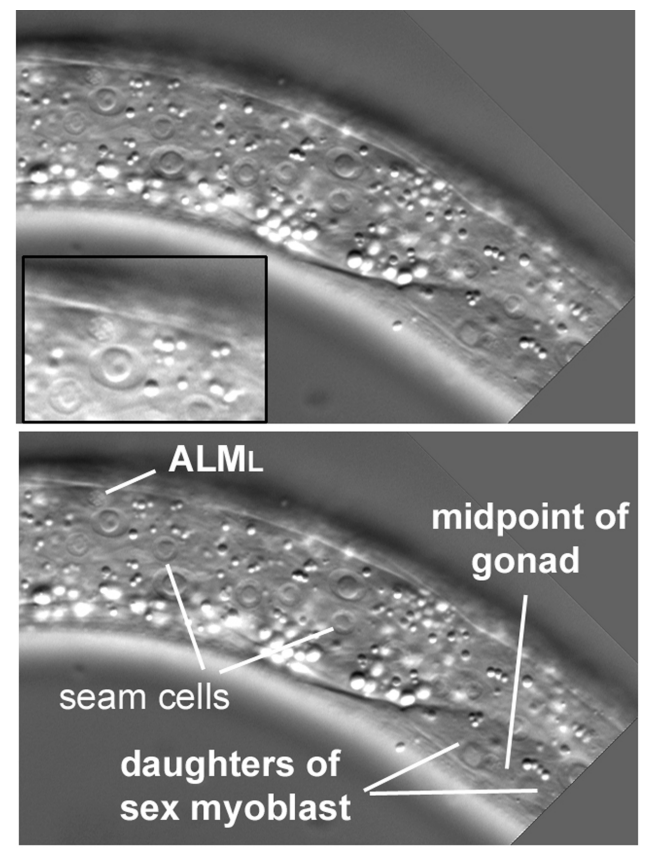

Figure 36. The isolated cell body of the neuron ALML dorsal to and anterior of the anteroposterior midpoint of the gonad.

\subsubsection{The central section}

The anterior part of the intestine occupies most of our view (Figure 37). One feature is part of the lumen of the intestine (the anterior part is out of the plane of focus). Often the lumen is distended with food that has been partly processed by the grinder of the pharynx, but the lumen is not particularly swollen in this animal. Two parallel tracks line the lumen. As for the small intestine of humans, the luminal (apical) surface of the $C$. elegans intestine consists of microvilli for maximum surface area. The appearance of the tracks is caused by the uniform length of the microvilli orthogonal to the long axis of the intestine and by the refractility of the plasma membranes of the densely packed microvilli.
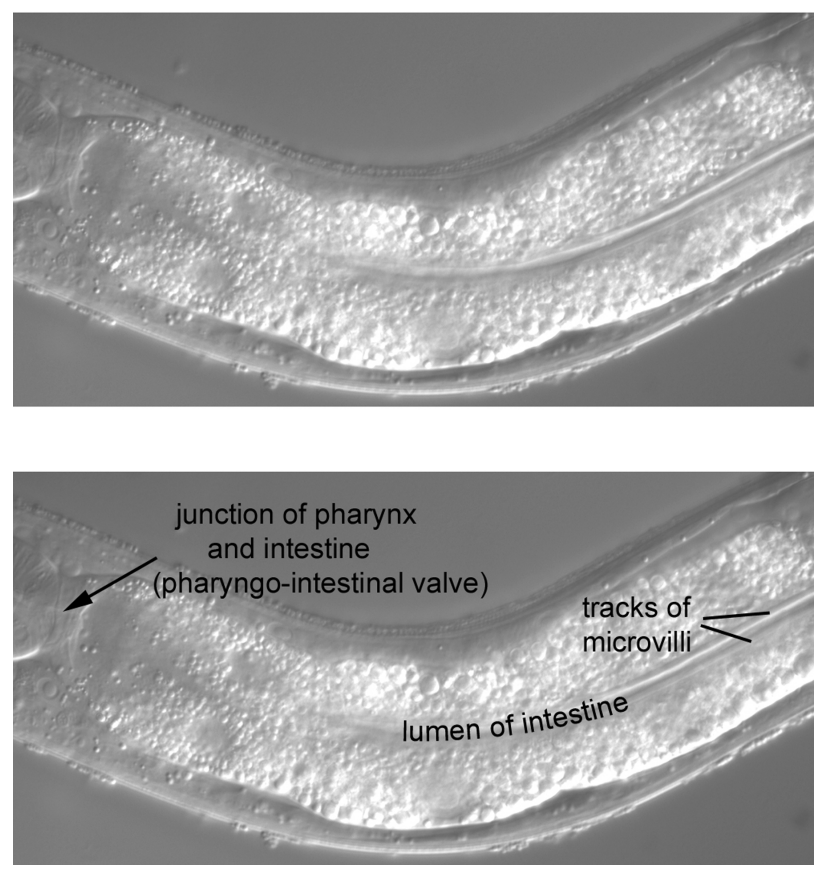

Figure 37. Parallel tracks of the intestinal microvilli in the central focal plane. 
A second feature is the junction of the pharynx and intestine, the pharyngo-intestinal valve, which is composed of the vpi cells (Figure 38). The nuclei of the valve are small, somewhat oddly shaped, and clearly posterior to the boundary with the pharynx (line drawings in upper right panel). Two of the nuclei are indicated in the Figure, as is the nucleus of $\mathrm{m} 8$, a muscle cell at the extreme posterior end of the pharynx. Useful for mosaic analysis, $\mathrm{m} 8$ and the vpi nuclei descend from MS. Note that the boundary of the valve with the intestine is very clear in this image (arrowheads).
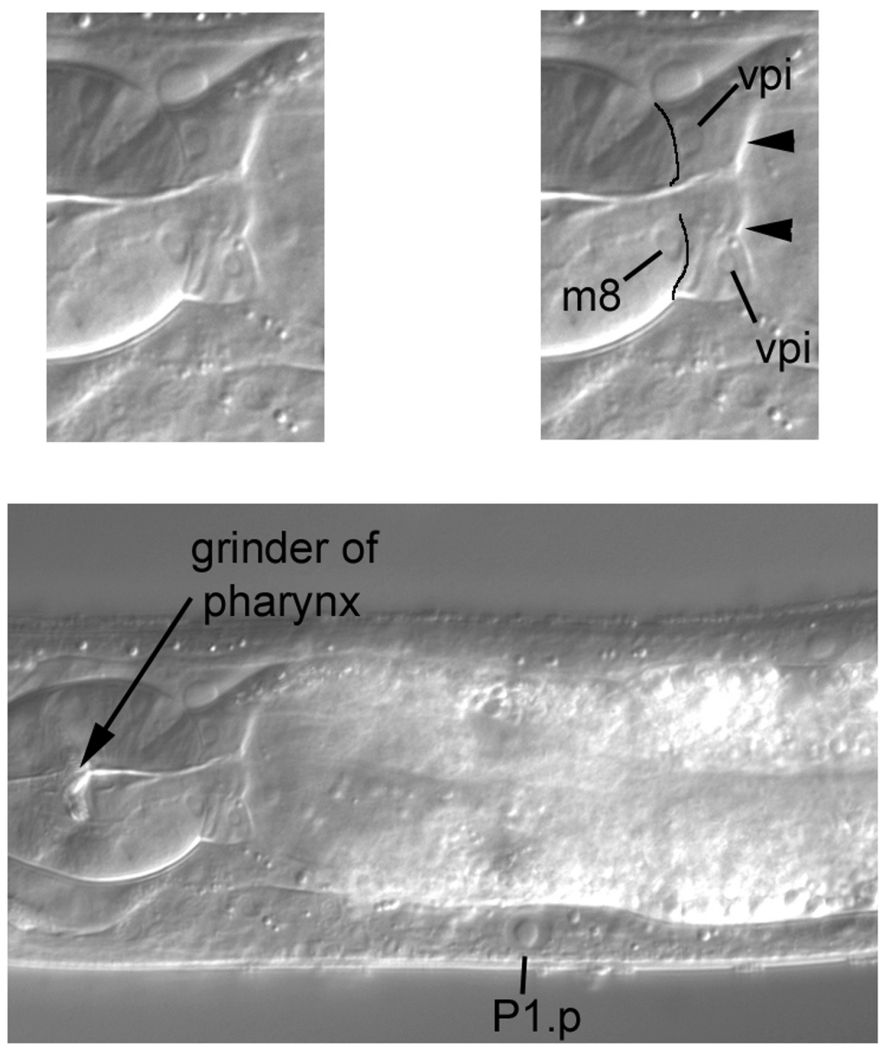

Figure 38. Several vpi nuclei in the pharyngo-intestinal valve.

\subsection{The head}

It is not possible here to do justice to the rich complexity of the head. The pharynx and nervous system are challenges in their own right, but there are also body muscles, hypodermal nuclei, and the nuclei of the excretory system. We shall, nevertheless, keep to our goal of pointing out a few landmarks which bear on each of these cell types. With these landmarks, one should be able to learn more by consulting the published diagrams and computer resources. With this approach, many of the neurons of the ganglia of the head can be identified, even though we shall only show a few. The pharynx will be discussed briefly at the end of the tutorial. More Nomarski images of the pharynx can be viewed at an on-line site from Leon Avery (see the Pharynx Atlas), and diagrams of the positions of the pharyngeal nuclei have been published (Bargmann and Avery, 1995). 


\subsection{The posterior half of the head}

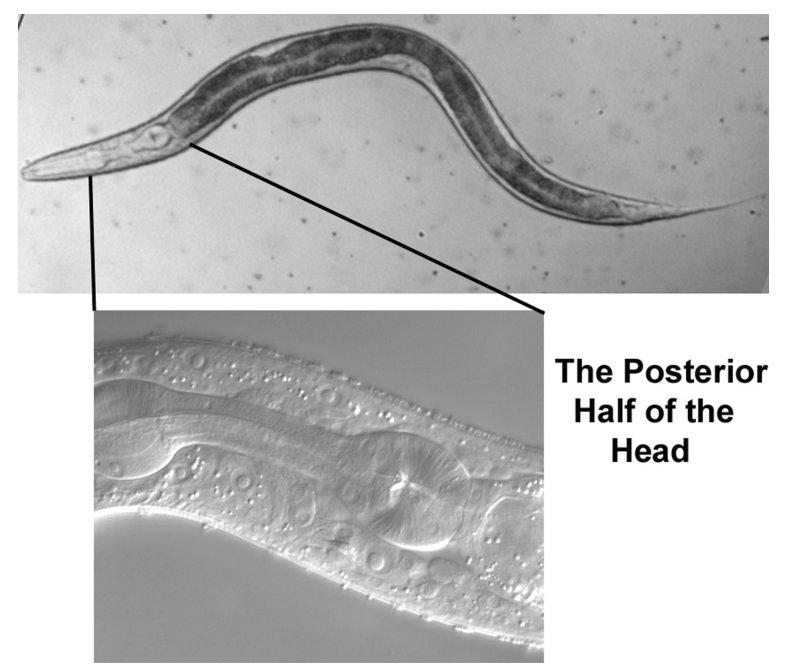

Figure 39.

\subsubsection{Left lateral sections}

Our first image of the left side contains hyp7 and seam-cell nuclei, as has been the case all along our journey from the posterior to the anterior of the worm (Figure 40). As a reference for anteroposterior position in this part of the head, the locations (not visible in this focal plane) of the anterior end of the intestine, of the pharyngeal metacorpus (the anterior bulb about half way along the length of the pharynx), and of the terminal or posterior bulb are indicated. We shall, of course, encounter them as we move into the central longitudinal sections. Note the two small nuclei-one is bigger than the other-which are located very laterally. These are the sheath and socket cells for the left anterior deirid (ADEL) sensory neuron whose dendrite enters the cuticle in this region [recall the nucleus of the postdeirid sheath cell in the lateral section of the posterior part of the middle body (Figure 21)]. 

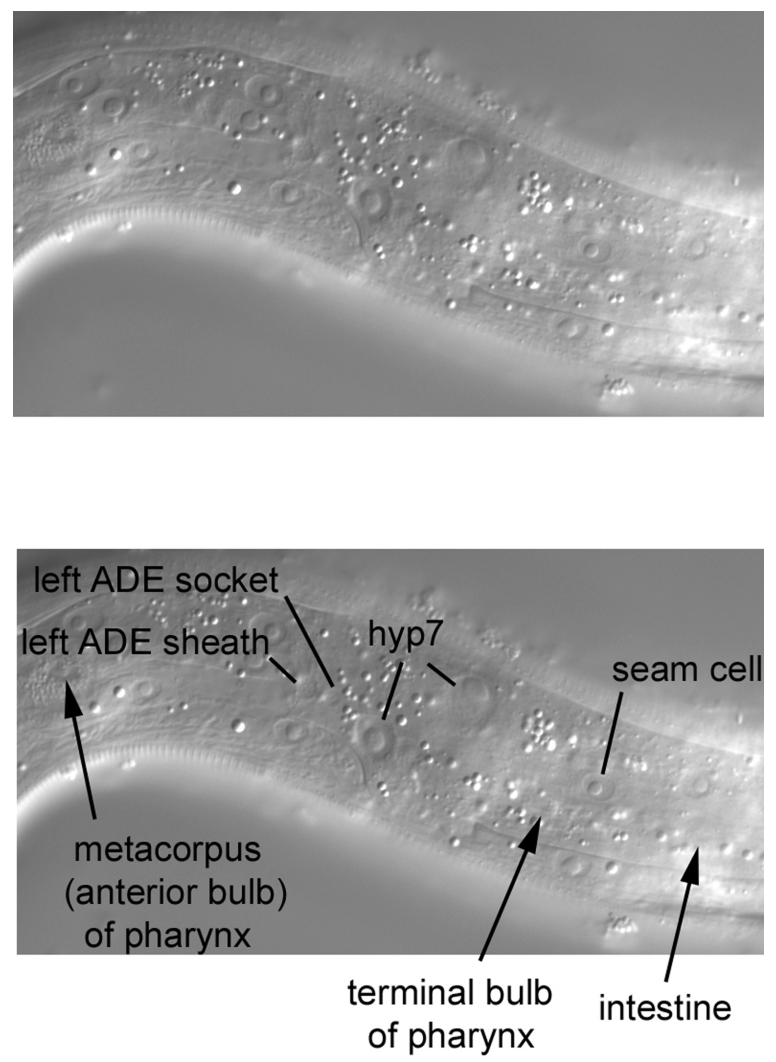

Figure 40. The far left side of the posterior half of the head.

Moving the focus an increment (the image is from a different L4 larva) brings many body muscles into view (Figure 41). Note how their nuclei are smaller than those from the middle part of the body. Although the nuclei here have the elliptical shape characteristic of body muscles, the potential exists to confuse them with the nuclei of hypodermal cells that are located both ventrally and dorsally in the central focal plane of the head. The drill of body muscle-central hypodermal nuclei-body muscle therefore very much applies to the head. Useful for mosaic analysis, the body muscles of the head derive from the blastomeres MS and D. 

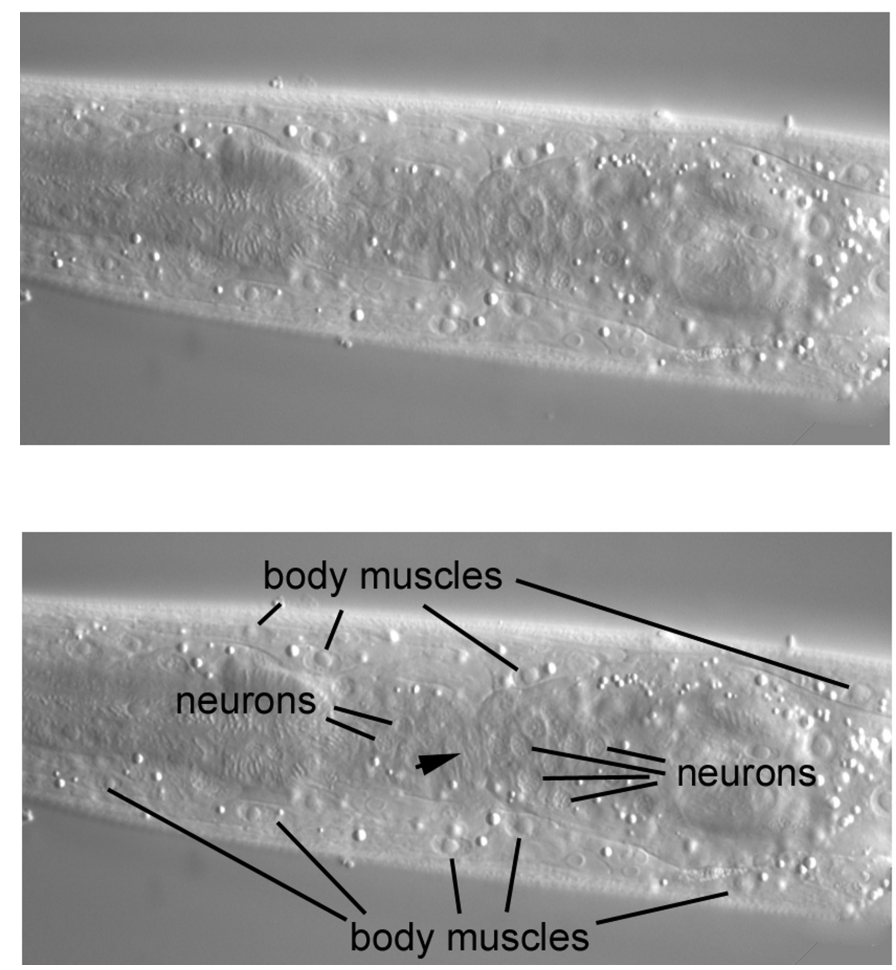

Figure 41. Moderately left, with body muscle and a few of the many neuronal nuclei.

Most of the neuronal cell bodies of the animal are concentrated around the isthmus of the pharynx, the long, thin section between the metacorpus and the terminal bulb. Visible laterally are two clusters of several of these cell bodies. Note that the clusters are separated by a region of what appears to be dorsal-ventral striations (arrowhead). This is the left lateral part of the nerve ring, a bundle of neuronal processes that surrounds the isthmus.

Moving the focus again brings more of the pharynx into view (some of the pharyngeal nuclei are indicated with arrowheads; Figure 42). Of particular note is a large nucleus in the dorsal region just anterior to the terminal bulb of the pharynx. It has a large nucleolus and is clearly separated from the body wall; it appears instead to belong to the same tissue as do neighboring neuronal nuclei just ventral to it. This is the nucleus of the left amphid sheath cell. There is a contralateral partner on the right. These sheath cells are support cells for the amphid sensory neurons. The left sheath cell sends a process to the left side of the anterior tip of the animal; the amphid dendrites from the left side are embedded in the anterior end of this process at their junction with the body cuticle. 

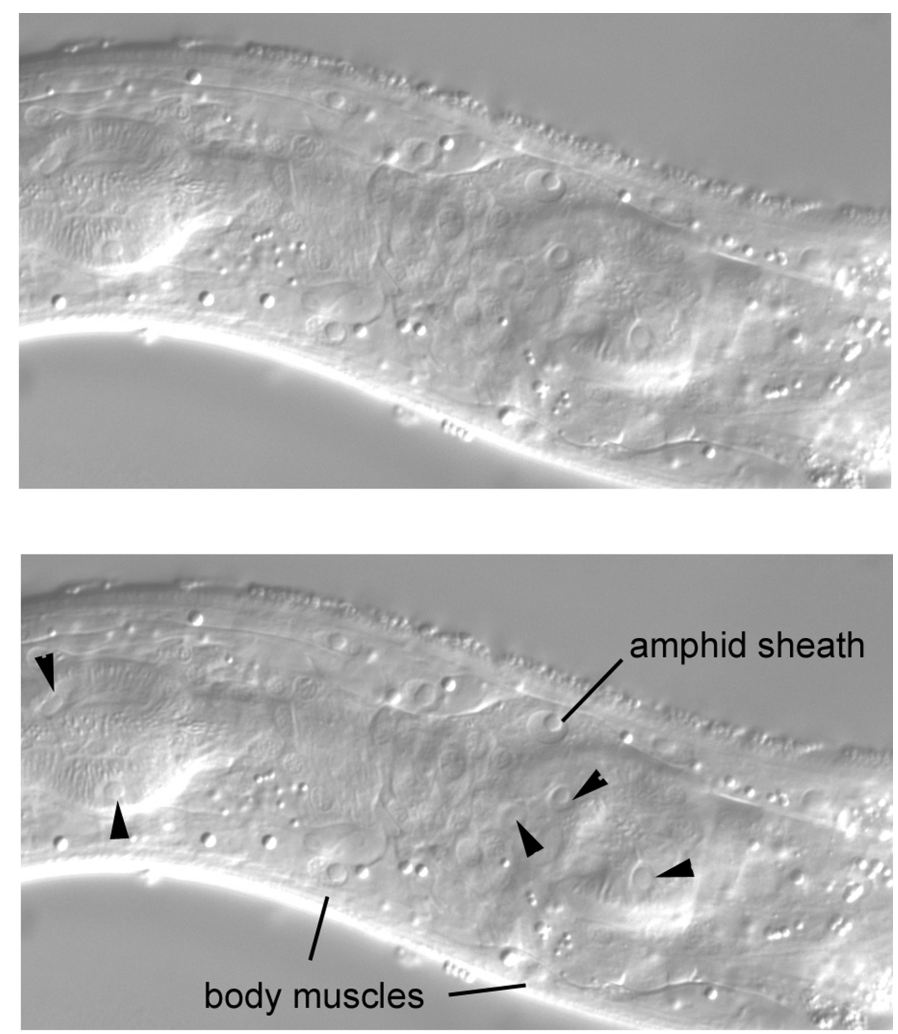

Figure 42. Still left, with the nucleus of the amphid sheath cell.

Another move of the focus brings us almost but not quite to the central longitudinal section. More of the pharynx is in view (some of the nuclei of the pharynx will be studied at the end of the tutorial; Figure 43). Also, the large nucleus of the excretory cell, which is a landmark for the central section, is just coming into view ventral to the terminal bulb of the pharynx. Of particular note here is an arc of three neuronal nuclei in the dorsal region. These are the cell bodies of ASKL, ADLL, and ASIL, which are three of the twelve amphid neurons. By returning to Figure 42, one can learn the close relationship of this arc of nuclei with the nucleus of the amphid sheath cell. These neurons are immediately left of and ventral to the sheath nucleus. The three nuclei have contralateral partners on the right side, but the cells on the right are not necessarily lineal homologues of the cells on the left, which is important to keep in mind for mosaic analysis. Although ASKR, ADLR, ASIL, and ASIR descend from the ABp blastomere (the ASI neurons are actually left-right lineal homologues), ASKL and ADLL descend from ABa. 

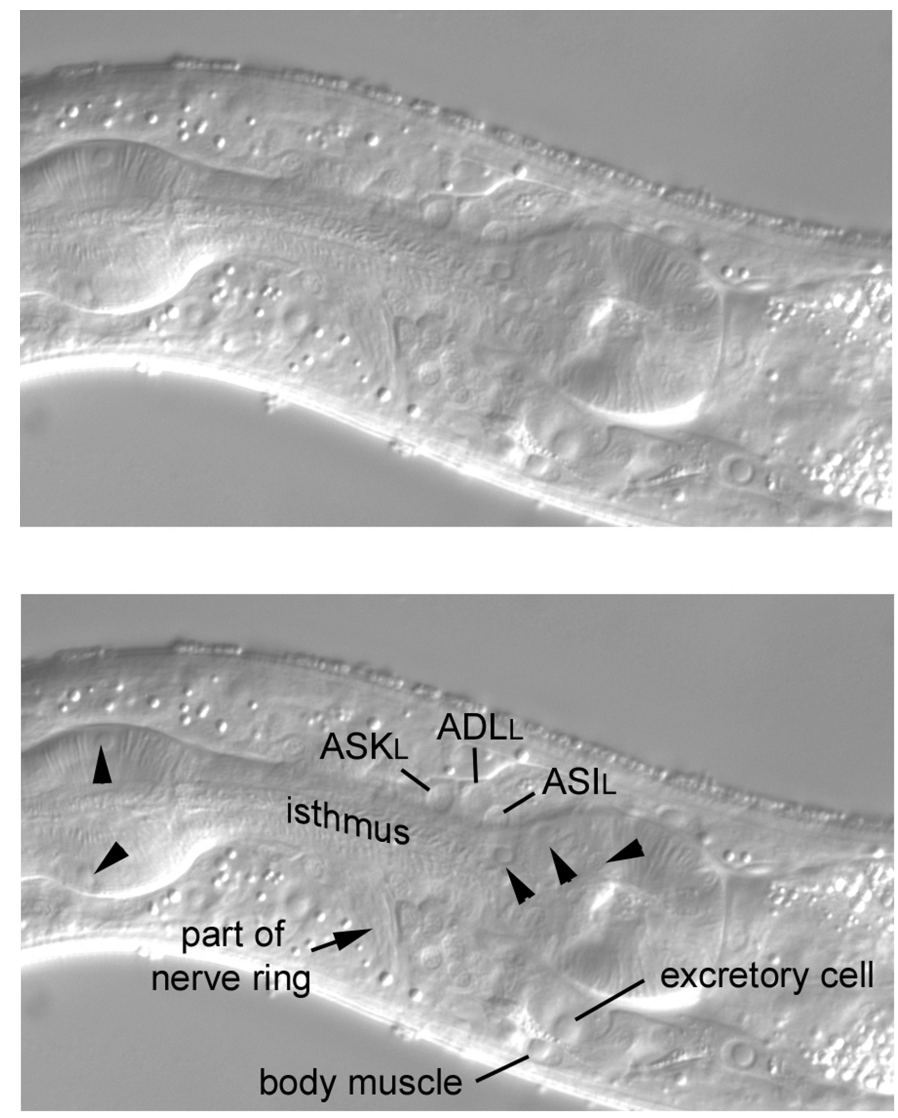

Figure 43. Slightly left, an arc formed by the nuclei of three of the amphid neurons.

\subsubsection{The central sections}

In Figure 44, the body muscle is one clue that we have yet to reach the central focal plane. (Among other clues, the grinder of the pharynx and the nucleus of the excretory cell are not completely in the focal plane.) We are lingering just to the left of the central plane in order to view one of the two gland cells of the excretory system (the other one is contralateral). The nucleus is at the posterior end of the cell, and the cytoplasm highly granular. A projection, which is easily seen because of its granularity (arrowheads), is sent to the region just anterior of the nucleus of the excretory cell. 

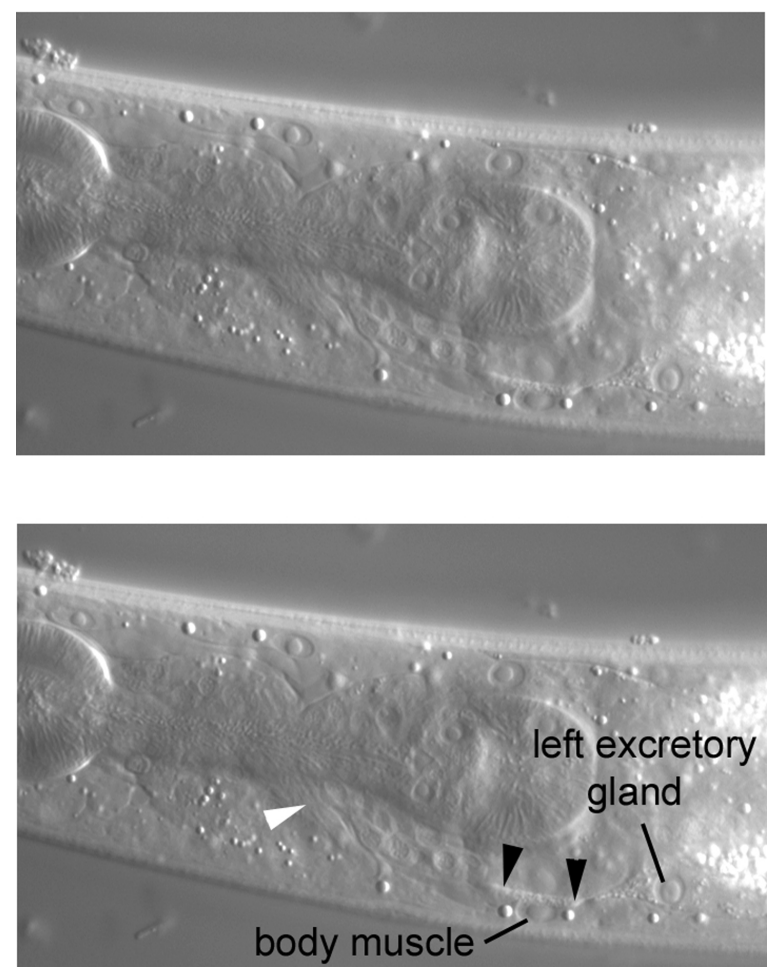

Figure 44. Near the central focal plane, the nucleus and anterior process of the left excretory gland.

A nucleus called G2.p comes into view with a slight move of the focus towards the central section (Figure 45). In this L4 larva, it is also part of the excretory system, being the pore or socket cell at the junction of the excretory duct and the body cuticle. Earlier in development, G2.p is not the socket, but by the middle of the L2 stage, it permanently assumes this function. 

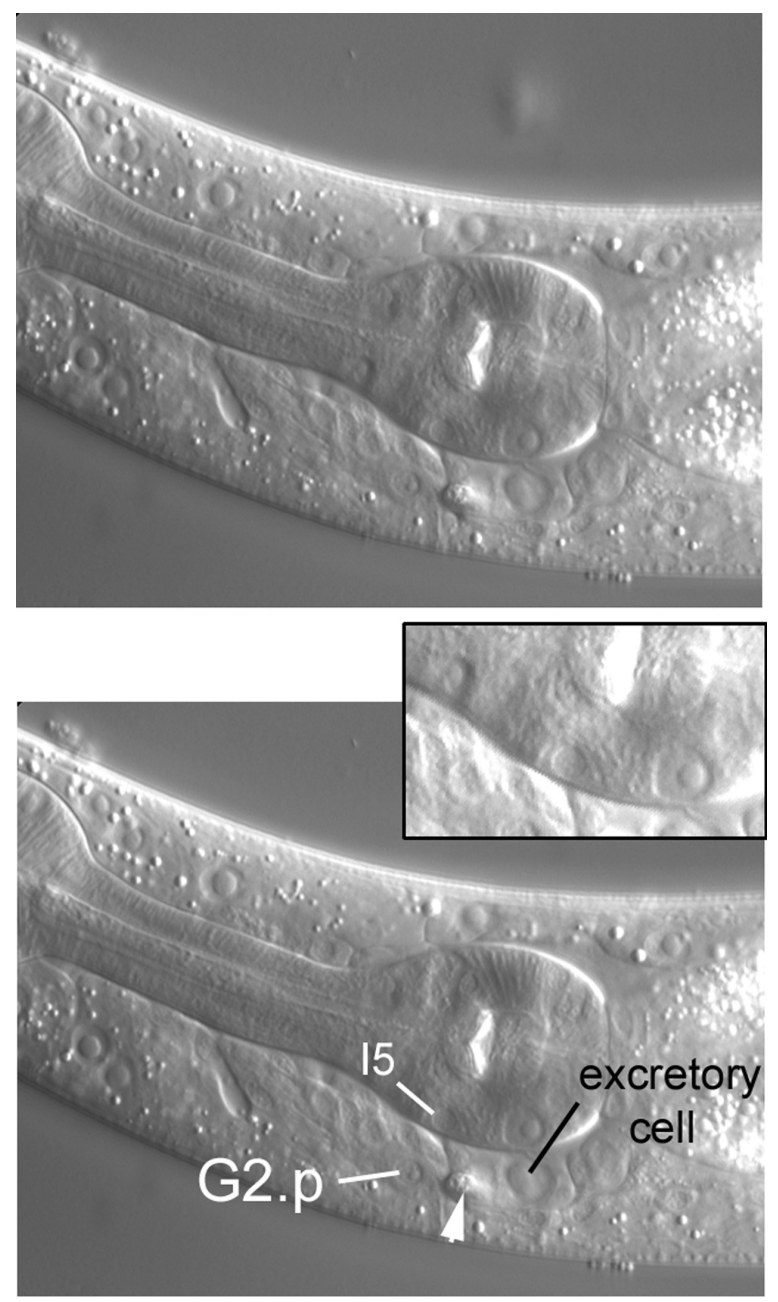

Figure 45. Nearer the central focal plane, the nucleus of G2.p, in this animal the likely excretory socket cell. Inset, I5, a pharyngeal neuron mentioned later.

With a move into the central focal plane (Figure 46), the nucleus of the excretory cell is now prominent and unambiguous. Just anterior to it is the small, elliptical nucleus of the excretory duct cell. The duct itself, connecting the lumen of the excretory cell to the external environment, is evident (arrowheads). Note that the duct in another animal is visible in Figure 39. Recall that we were able to see in Figure 32 the left posterior canal of the H-shaped excretory cell. The two anterior canals and the two posterior canals of this cell ascend dorsally and laterally from the cell body containing the nucleus and extend for nearly the entire anterior or posterior lengths of the animal. The excretory system is important for osmoregulation (Nelson and Riddle, 1984). Being a bag of concentrated solutes, the animal must remove excess water that diffuses in. Removal might be achieved by means of the secretion of $\mathrm{K}^{+}$, $\mathrm{Na}^{+}$, and $\mathrm{Cl}^{-}$into the lumen of the canals of the excretory cell. The solutes and water move through the canals and out of the animal via the duct. 

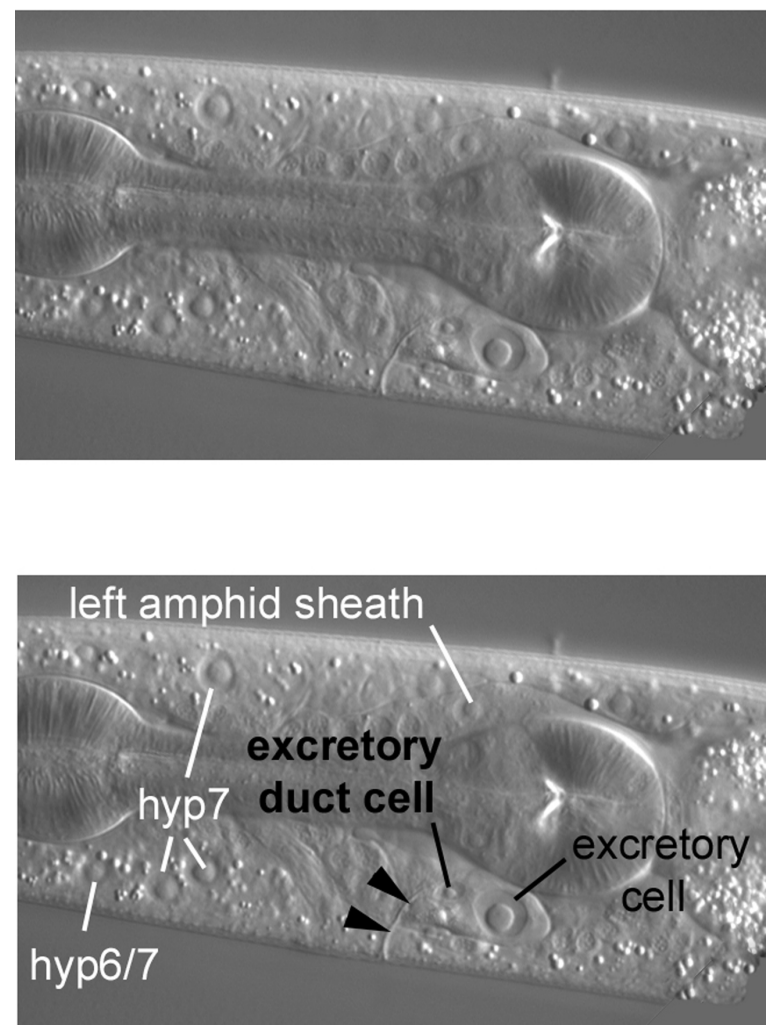

Figure 46. The nucleus of the excretory duct cell anterior of the prominent nucleus of the excretory cell. Arrowheads, the excretory duct, made refractile by its cuticle.

Set off from other nuclei just dorsal to the isthmus of the pharynx in the central plane are three neuronal nuclei that are easy to identify (Figure 47). The nucleus of the anterior-most cell, RMED, is elliptical and ascends dorsally from anterior to posterior. The nuclei of the other two, RID and ALA, are round, and ALA is noticeably the largest of the three. These cells descend from ABa and are useful for mosaic analysis. Processes from RID and ALA are important components of the dorsal nerve cord. The ALA nucleus can in turn be used to locate CEPDL or CEPDR left or right of it. 

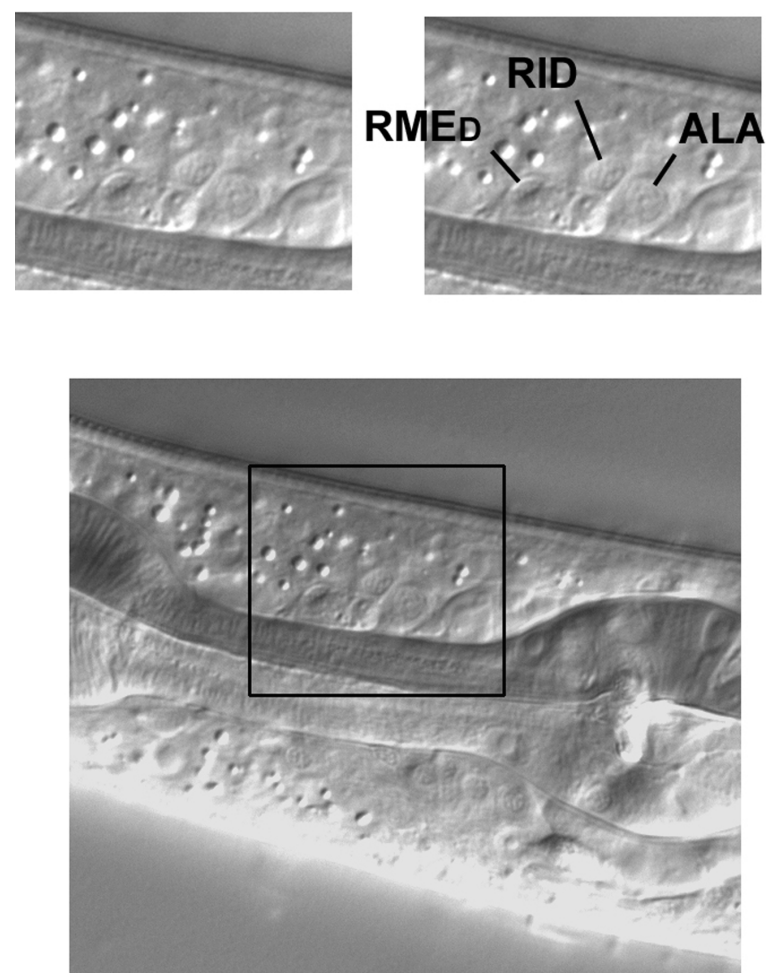

Figure 47. Three neuronal nuclei from $\mathrm{ABa}$ in the central focal plane dorsal of the pharyngeal isthmus.

\subsection{The anterior half of the head}

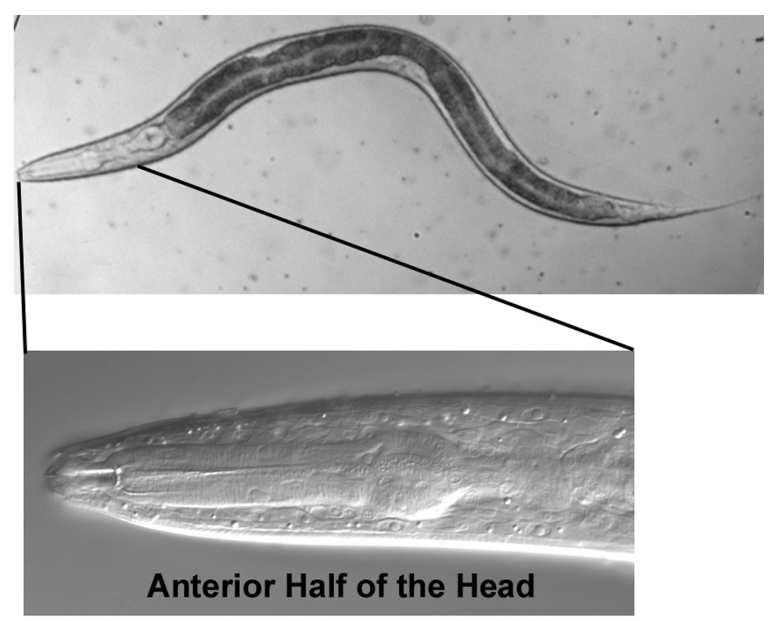

Figure 48.

\subsubsection{Left lateral sections}

The upper two panels of Figure 49 exhibit lateral hypodermal nuclei near the tapered anterior end of the animal (in the bottom panel, a corresponding view of the pharynx provides an anteroposterior frame of reference). H0L, the anterior-most seam cell, is the only seam cell that does not divide postembryonically. Hyp5 is a binucleate cell (the other nucleus is contralateral) that forms a cylindrical part of the hypodermis.Moving the focus towards the central focal plane (bottom panel), we encounter body muscles from the ventral and dorsal quadrants on the left side. Again, they can be confused with centrally located hypodermal nuclei. 

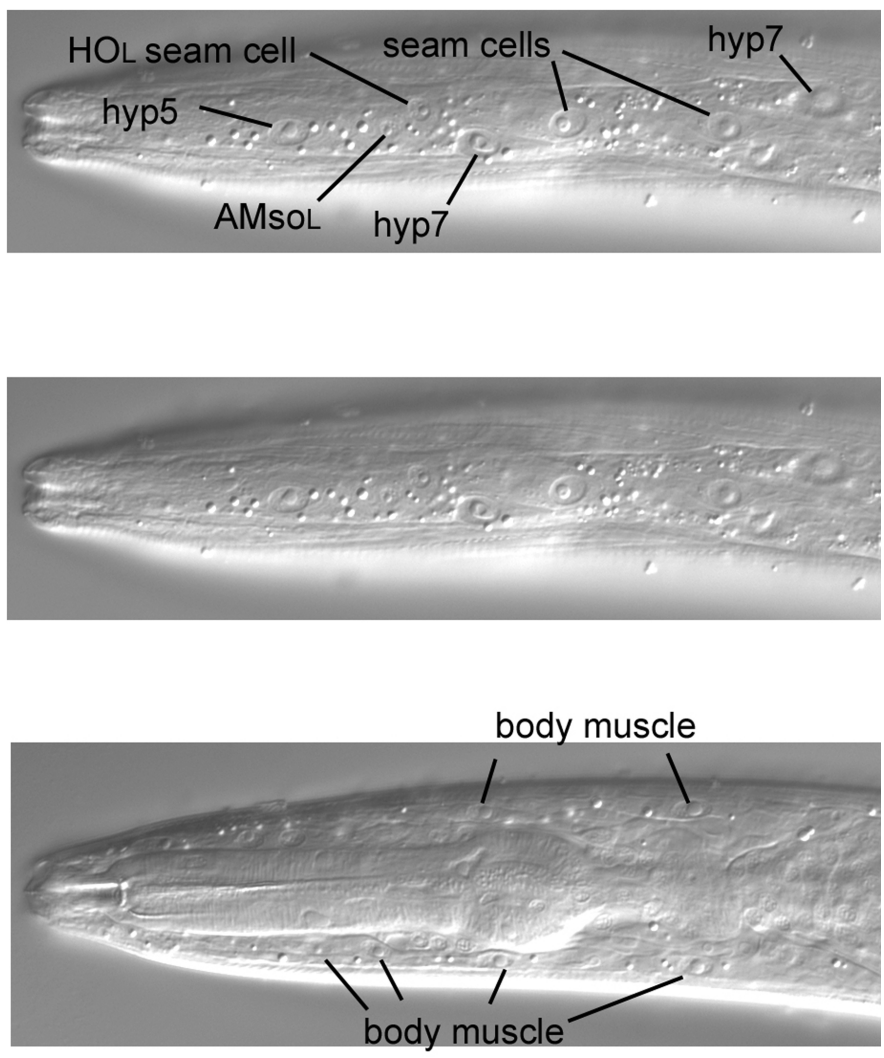

Figure 49. Hypodermal nuclei in the far left plane, and body muscles in the moderately left plane.

\subsubsection{The central sections}

The dorsal hypodermal nuclei in the central longitudinal section are shown in Figure 50. In the top panel, the four nuclei labeled with an asterisk are four of the six nuclei (the other two are ventral) that join hyp7 during larval development (Yochem et al., 1998). From birth until the late L2 stage, these six nuclei compose a separate syncytium called hyp6. In the L4 larva shown here, these four nuclei are part of hyp7. Other dorsal hypodermal nuclei are evident in the lower panels. The anterior-most nucleus shown is one of the three that compose hyp4 (the other two are ventral, as we shall see). This nucleus is elliptical and easily distinguished from the two hyp3 nuclei posterior to it. Hyp3 is binucleate, and the two nuclei clearly resemble each other. 

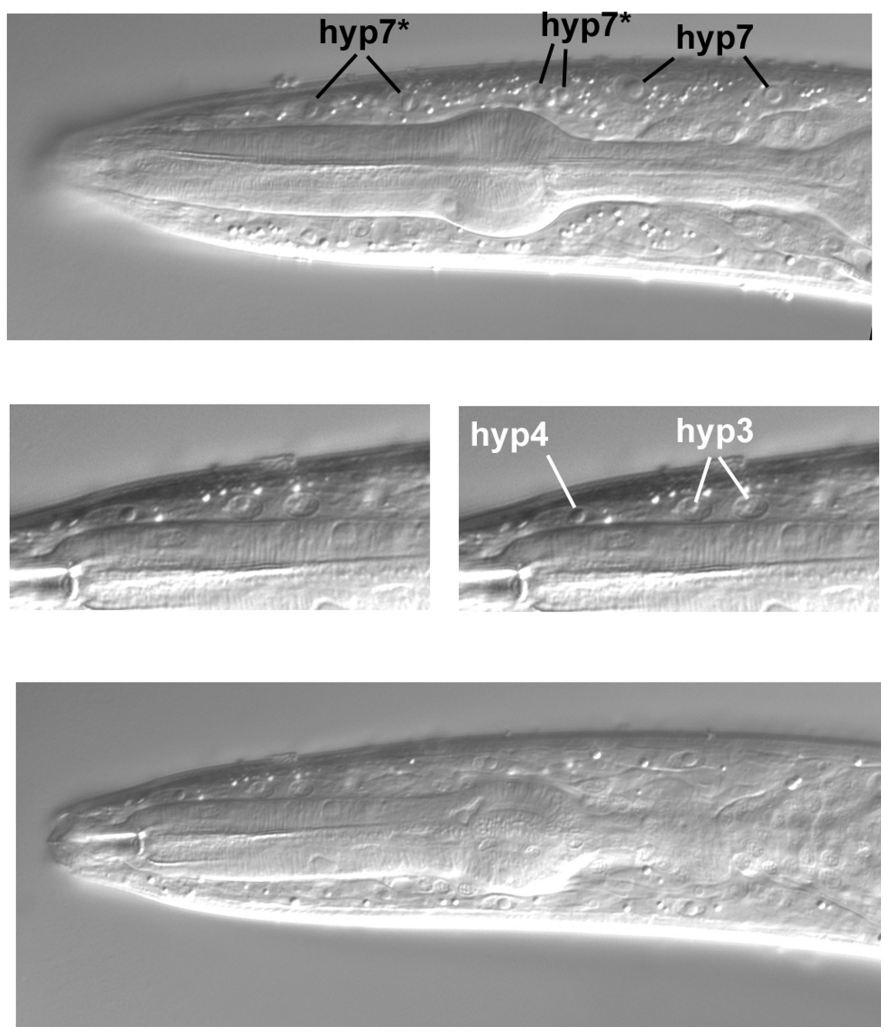

Figure 50. Dorsal hypodermal nuclei in the central focal plane.

The two ventral hyp6 nuclei that are now part of hyp7 are indicated in the upper panels of Figure 51. The posterior hyp7* nucleus is usually just anterior to a pair of natal hyp7 nuclei. As a reminder that reality is often opposed to text-book images, the focus had to be changed slightly in order to bring the anterior hyp $7 *$ nucleus into view (middle panel). Note the relatively large size of this nucleus and that there is an absence of body muscles in its vicinity, reinforcing our conclusion that we are truly in the central plane of this part of the worm. For comparison, note in the top panel the small nucleus of a body muscle slightly anterior to the corresponding anteroposterior position of the hyp $7^{*}$ nucleus. (In truth, this worm was moving its head slightly, thereby distorting the focal planes, but we decided to take images anyway.) Another adjustment of the focus was required to bring the two ventral nuclei of hyp4 into view (lower panel and inset). Note their small size and the absence of nuclei anterior of them in this focal plane. 

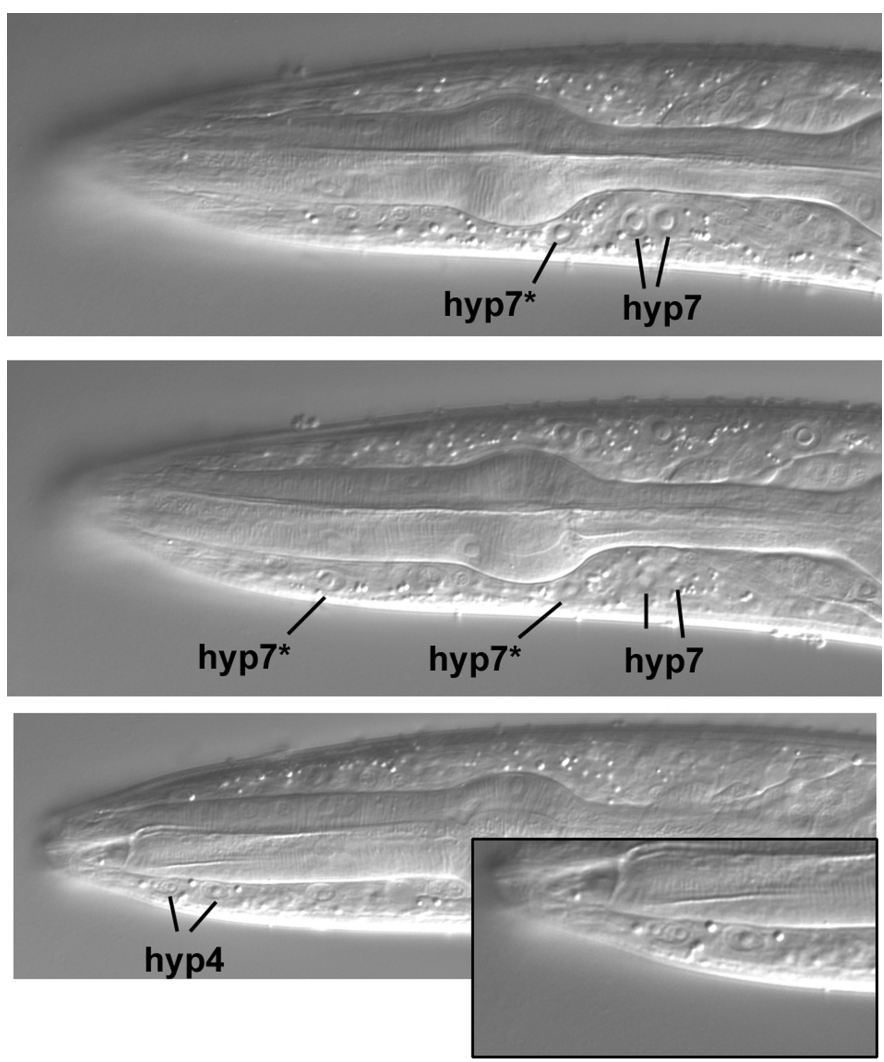

Figure 51. Ventral hypodermal nuclei in the central focal plane.

\subsection{A brief view of the pharynx}
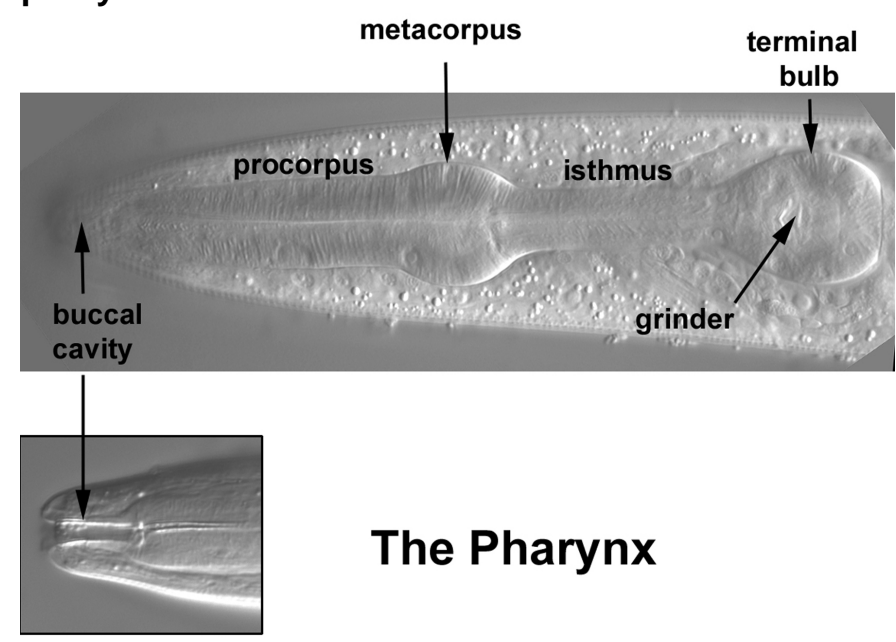

\section{The Pharynx}

Figure 52.

The pharynx is composed of nuclei that descend only from ABa and MS. In contrast to the simple left-right symmetry of the body plan, the pharynx has a triangular organization (Albertson and Thomson, 1976). With implications for mosaic analyses, some of the cells are syncytial. This is especially true for the muscle cells, but some, such as $\mathrm{m} 8$, the posterior-most nucleus of the pharynx which we saw in Figure 38, are mononucleate. The nuclei of the pharynx are fun to study, but only a few will be presented here (more micrographs can be added later if there is interest). Nevertheless, the few shown should serve as good landmarks for learning every nucleus based on the published diagrams and PharynxAtlas. 


\subsubsection{Left lateral sections}

Two of the left-most nuclei of $\mathrm{m} 3$, a designation for three binucleate muscle cells in the procorpus, are shown (Figure 53), as is one of the $\mathrm{m} 4$ nuclei. Note the oblong shape of the two $\mathrm{m} 3$ nuclei (nearly rectangular with jagged edges), which make finding them unambiguous; there are no other nuclei like them in the pharynx. This is not the extreme left view. If we were to go further left, the nucleus of $\mathrm{m} 4 \mathrm{~L}$, which has an unusual shape, would be visible in the exact center of the left-most side of the metacorpus.
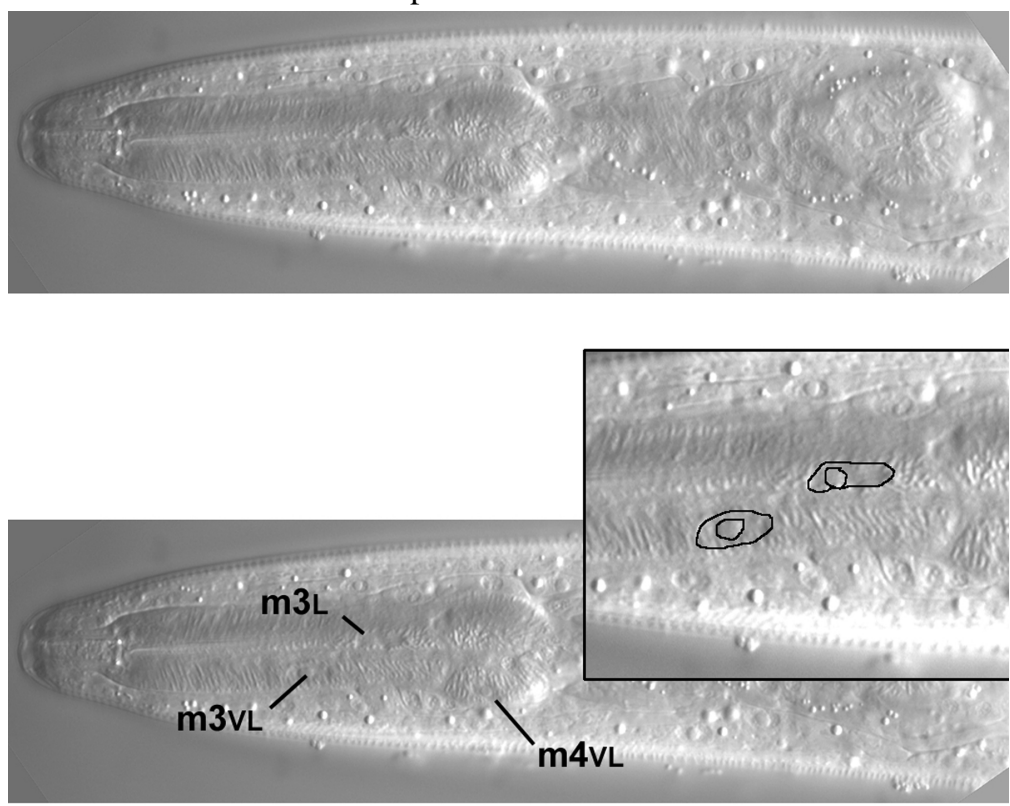

Figure 53. A few of the left lateral nuclei of the pharynx.

Moving the focus towards the central focal plane, the left dorsal $\mathrm{m} 3$ nucleus is now in view (Figure 54). The nucleus of I5, one of several neurons in the pharynx, is becoming visible. I5 can also be seen in Figure 45 and its inset. Note outside the pharynx a nice view of the left amphid sheath cell and the arc of three amphid neurons.
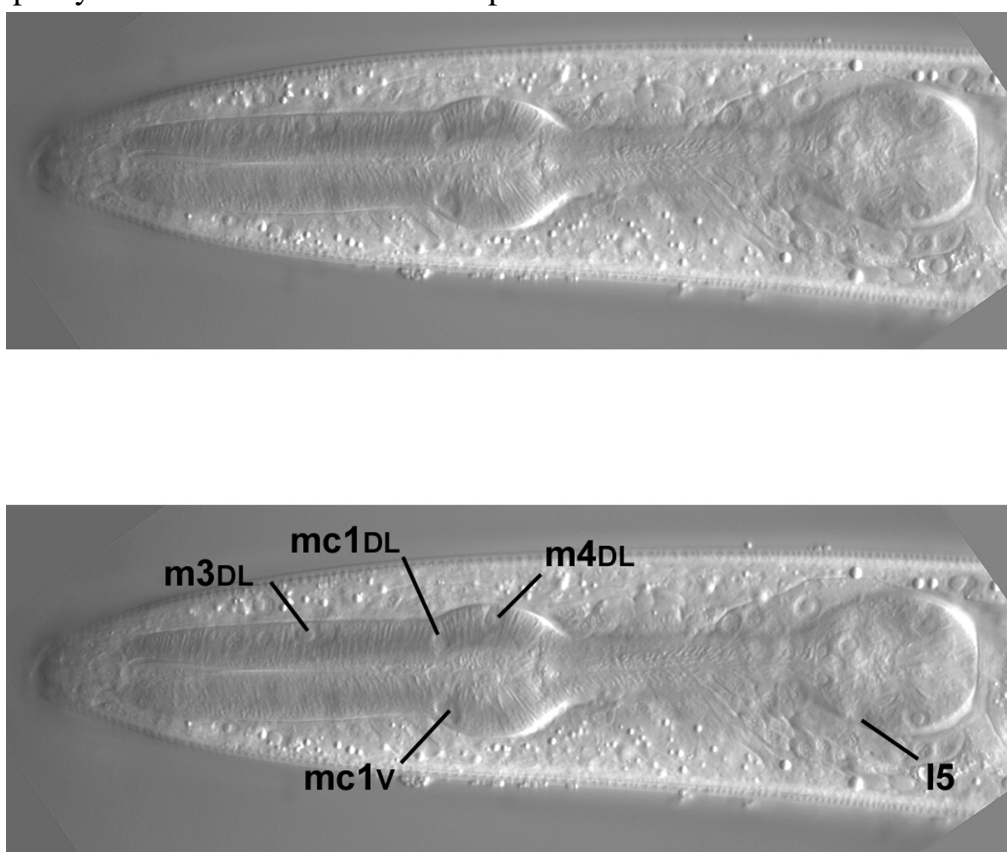

Figure 54. A few pharyngeal nuclei closer to the central focal plane. 


\subsubsection{The central sections}

I5, which descends from ABa, is now more in focus (Figure 55). At the junction of the isthmus and terminal bulb are two of three mc2 nuclei (all of them descend from ABa). They often have an unusual, noncircular shape. The focal plane of this image is not perfectly central, because the m3VR nucleus, which is contralateral to m3VL, is just visible in the procorpus. On this imperfect note, a glimpse of the m3VR nucleus from the right fringe, the tutorial ends.
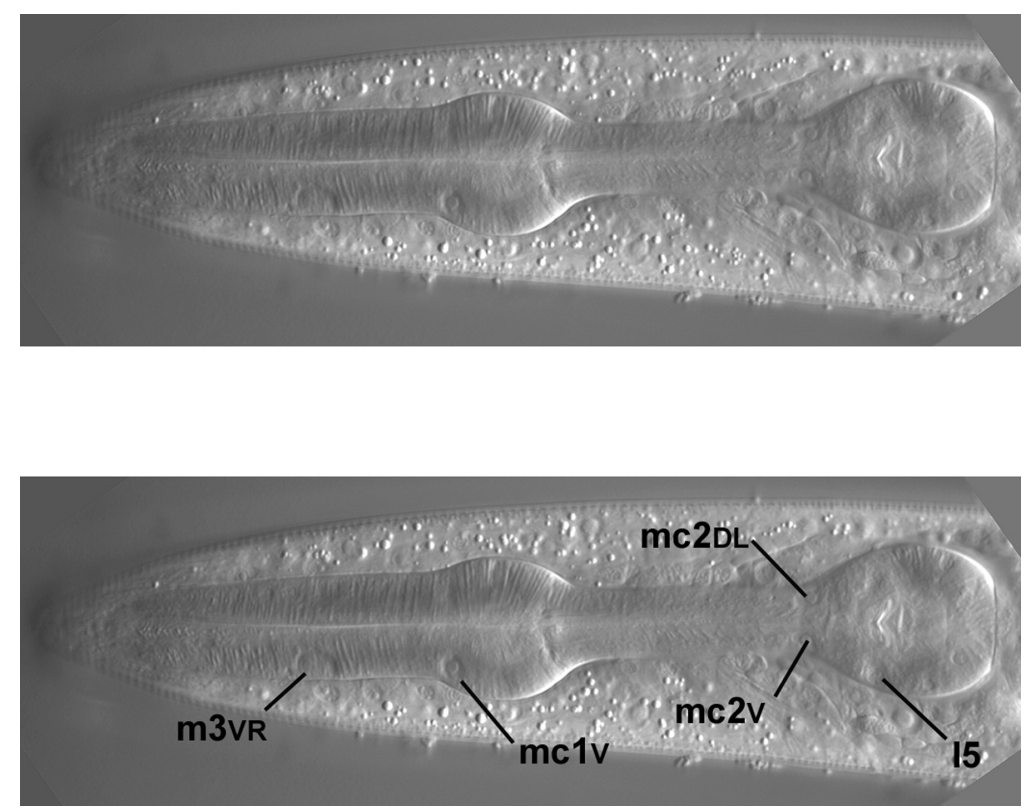

Figure 55.

\section{Acknowledgments}

Much gratitude is extended to Leslie Bell, for her comments and suggestions; to Meera Sundaram, for sharing her knowledge of the anatomy of C. elegans; to Lihsia Chen, for sharing her microscope; and to Robert Herman, for his generous support.

\section{References}

Albertson, D.G., and Thomson, J.N. (1976). The pharynx of Caenorhabditis elegans. Philos. Trans. R. Soc. Lond., B, Biol. Sci. 275, 299-325. Abstract

Bargmann, C.I., and Avery, L. (1995). Laser killing of cells in Caenorhabditis elegans. Methods Cell Biol. 48, 225-250. Abstract

Francis, R., and Waterston, R.H. (1991). Muscle cell attachment in Caenorhabditis elegans. J. Cell Biol. 114, 465-479. Abstract Article

Hedgecock, E.M., and Herman, R.K. (1995). The ncl-1 gene and genetic mosaics of Caenorhabditis elegans. Genetics 141, 989-1006. Abstract

Kenyon, C. (1986). A gene involved in the development of the posterior body region of C. elegans. Cell 46, 477-487. Abstract Article

Kimble, J., and Hirsh, D. (1979). The postembryonic cell lineages of the hermaphrodite and male gonads in Caenorhabditis elegans. Dev. Biol. 70, 396-417. Abstract Article 
Köppen, M., Simske, J.S., Sims, P.A., Firestein, B.L., Hall, D.H., Radice, A.D., Rongo, C., and Hardin, J.D. (2001). Cooperative regulation of AJM-1 controls junctional integrity in Caenorhabditis elegans epithelia. Nat. Cell Biol. 3, 983-991. Abstract Article

Lackner, M.R., Kornfeld, K., Miller, L.M., Horvitz, H.R., and Kim, S.K. (1994). A MAP kinase homolog, mpk-1, is involved in ras-mediated induction of vulval cell fates in Caenorhabditis elegans. Genes Dev. 8, 160-173. Abstract

Miller, L.M., Waring, D.A., and Kim, S.K. (1996). Mosaic analysis using a ncl-1 (+) extrachromosomal array reveals that lin-31 acts in the Pn.p cells during Caenorhabditis elegans vulval development. Genetics 143, 1181-1191. Abstract

Moribe, H., Yochem, J., Yamada, H., Tabuse, Y., Fujimoto, T., and Mekada, E. (2004). Tetraspanin protein (TSP-15) is required for epidermal integrity in Caenorhabditis elegans. J. Cell Sci. Pt. Abstract Article

Myers, T.R., and Greenwald, I. (2005). lin-35 Rb acts in the major hypodermis to oppose ras-mediated vulval induction in C. elegans. Dev. Cell 8, 117-123. Abstract Article

Nelson, F.K., and Riddle, D.L. (1984). Functional study of the Caenorhabditis elegans secretory-excretory system using laser microsurgery. J. Exp. Zool. 231, 45-56. Abstract Article

Sulston, J.E., and Horvitz, H.R. (1977). Post-embryonic cell lineages of the nematode, Caenorhabditis elegans. Dev. Biol. 56, 110-156. Abstract Article

Sulston, J.E., Schierenberg, E., White, J.G., and Thomson, J.N. (1983). The embryonic cell lineage of the nematode Caenorhabditis elegans. Dev. Biol. 100, 64-119. Abstract Article

White, J.G., Southgate, E., Thomson, J.N., and Brenner, S. (1986). The structure of the nervous system of the nematode Caenorhabditis elegans. Philos. Trans. R. Soc. Lond., B, Biol. Sci. 314, 1-340.

Yochem, J., Gu, T., and Han, M. (1998). A new marker for mosaic analysis in Caenorhabditis elegans indicates a fusion between hyp6 and hyp7, two major components of the hypodermis. Genetics 149, 1323-1334. Abstract

Yochem, J., Sundaram, M., and Bucher, E.A. (2000). Mosaic analysis in Caenorhabditis elegans. Methods Mol. Biol. 135, 447-462. Abstract

Zhang, S., Ma, C., and Chalfie, M. (2004). Combinatorial marking of cells and organelles with reconstituted fluorescent proteins. Cell 119, 137-144. Abstract Article

All WormBook content, except where otherwise noted, is licensed under a Creative Commons Attribution License 